\title{
Kinematics of the ribbon fin in hovering and swimming of the electric ghost knifefish
}

\section{Citation}

Ruiz-Torres, R., O. M. Curet, G. V. Lauder, and M. A. Maclver. 2014. Kinematics of the Ribbon Fin in Hovering and Swimming of the Electric Ghost Knifefish. Journal of Experimental Biology 217, no. 20: 3765-3766. doi:10.1242/jeb.113670.

\section{Published Version}

doi:http:10.1242/jeb.113670

\section{Permanent link}

http://nrs.harvard.edu/urn-3:HUL.InstRepos:30593570

\section{Terms of Use}

This article was downloaded from Harvard University's DASH repository, and is made available under the terms and conditions applicable to Other Posted Material, as set forth at http:// nrs.harvard.edu/urn-3:HUL.InstRepos:dash.current.terms-of-use\#LAA

\section{Share Your Story}

The Harvard community has made this article openly available.

Please share how this access benefits you. Submit a story.

\section{Accessibility}




\title{
CORRECTION
}

\section{Kinematics of the ribbon fin in hovering and swimming of the electric ghost knifefish}

\author{
Ricardo Ruiz-Torres, Oscar M. Curet, George V. Lauder and Malcolm A. Maclver
}

Two errors appeared in J. Exp. Biol. 216, 823-834.

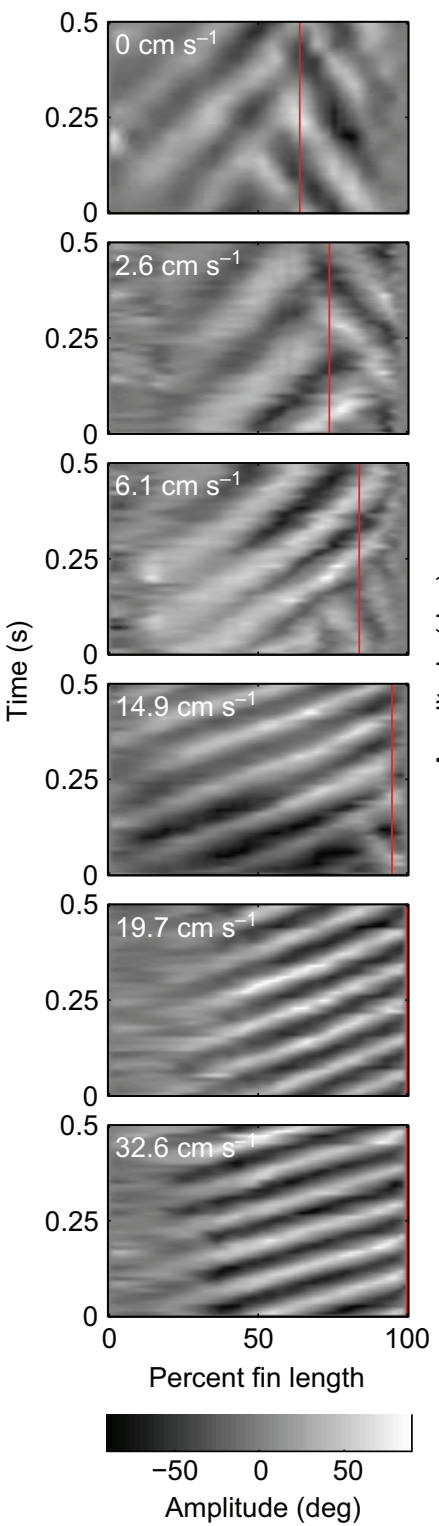

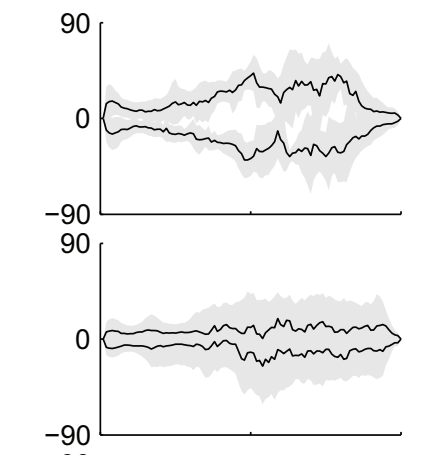
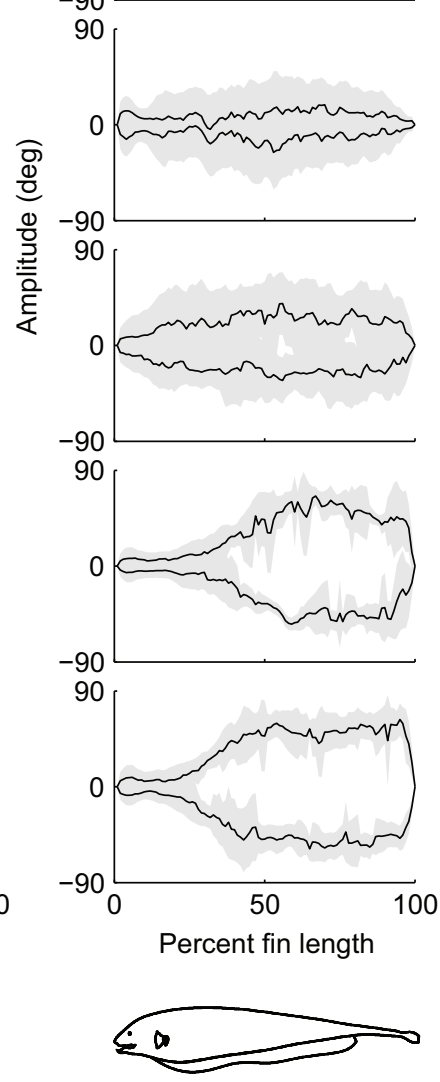

Fig. 2. Comparison of traveling wave amplitude patterns and wave envelope for different swimming velocities. Two counter-propagating waves are seen at low swimming velocities. The fraction of the fin occupied by the tail wave becomes shorter as the swimming velocity increases. Grayscale map is for amplitude in degrees. Estimated nodal point position for each swimming velocity is shown as a vertical red line. The first $500 \mathrm{~ms}$ of data are shown per trial for comparison. Trial duration varies amongst data sets. Mean wave envelope is shown in the right column. Gray area ranges vertically by \pm 1 s.d. of the peak magnitude of fin angle across the trial ( $N=530$ frames for $0 \mathrm{~cm} \mathrm{~s}^{-1}, N=1000$ for all other cases).

During data processing, the wrong scaling factor was used when converting the fin amplitude from pixels on the video to linear displacement in millimetres [term $y(x)$ in Eqn 1]. This error caused the angular displacement to be underestimated by a factor that varied between 2.9 and 3.6 (mean \pm s.d. $=3.28 \pm 0.27$ ) for the different data sets.

The error is found in Figs 2, 4, 9 and 10. In the right panels of Fig. 2, both panels of Fig. 4, Fig. 9C and the color bar in Fig. 10, the range of the $y$-axis is approximately three times smaller than it should have been.

In the Results and Discussion, we comment on the direction of the change in amplitude at different swimming speeds. The error does not affect these comments because the amplitude at all swimming speeds was scaled by approximately the same factor.

A second error was introduced when calculating the ratio of the fin length to the fin base length. The measurement of the fin base length was performed only on the first frame of every data set, instead of averaging the length across all the frames as was done for the fin length. This caused the ratio of fin length to fin base length to be larger in the hovering condition and smaller when swimming at $6 \mathrm{~cm} \mathrm{~s}^{-1}$. After the correction, the ratio is more constant than before.
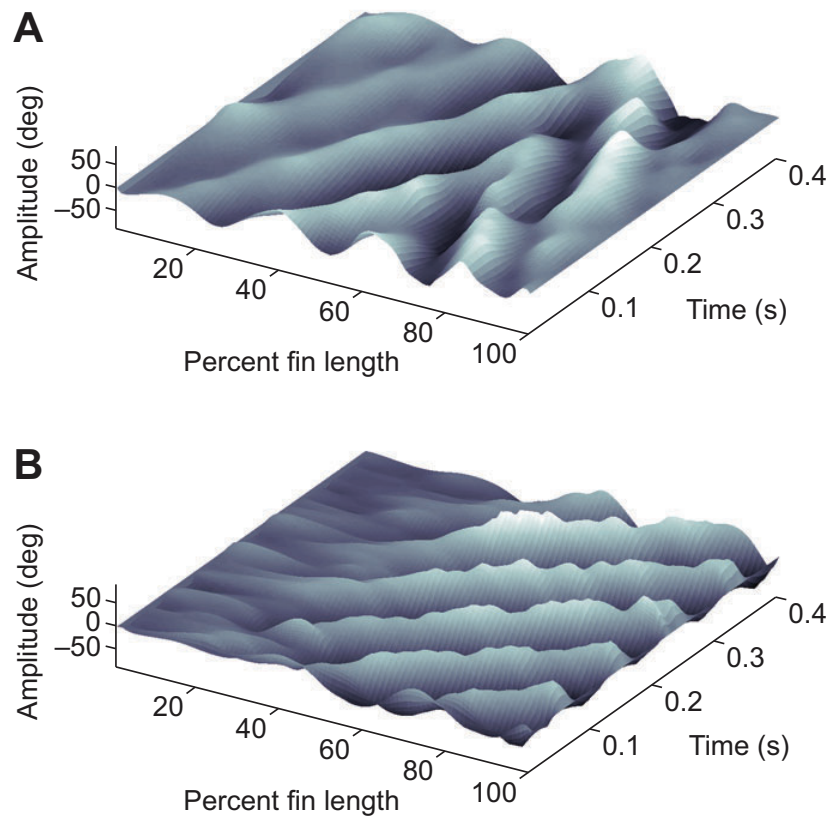

Fig. 4. Traveling wave comparison between (A) hovering and (B) surging at $19.7 \mathrm{~cm} \mathrm{~s}^{-1}$. A single wave travels caudally during forward swimming, while two waves traveling towards each other are observed while hovering. Grayscale is used to enhance amplitude information. The rostral end of the fin is at $0 \%$ on the fin length axis. 

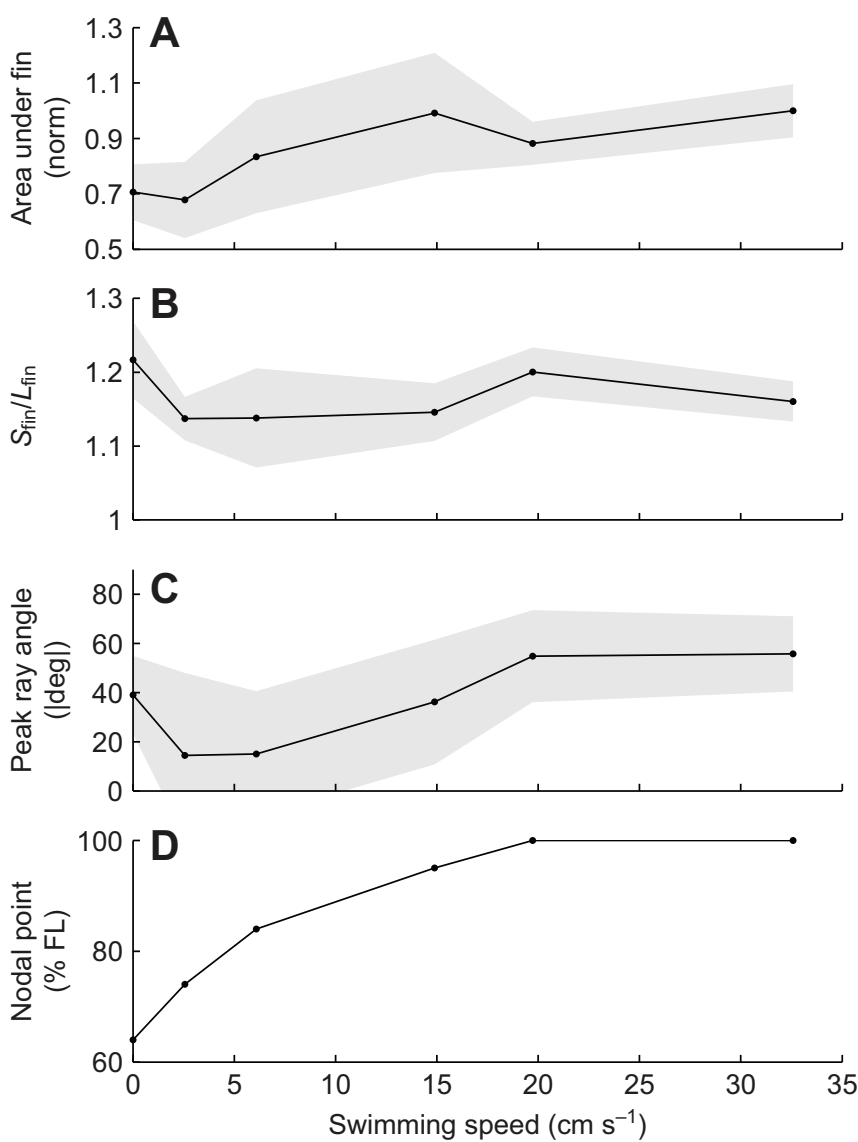

Fig. 9. Traveling wave variables as a function of swimming speed: (A) area under the fin, (B) normalized fin edge length, (C) peak ray angle and (D) nodal point location. The area under the fin is normalized with respect to the largest value. Fin edge length $\left(S_{\text {fin }}\right)$ is normalized with respect to the fin base length $\left(L_{\text {fin }}\right)$. Gray area ranges vertically by \pm 1 s.d. No s.d. is shown for nodal point location as this was obtained through visual inspection of the amplitude profiles (see Materials and methods). FL, fin length. $N=530$ frames for $0 \mathrm{~cm} \mathrm{~s}^{-1}, N=1000$ for all other cases.

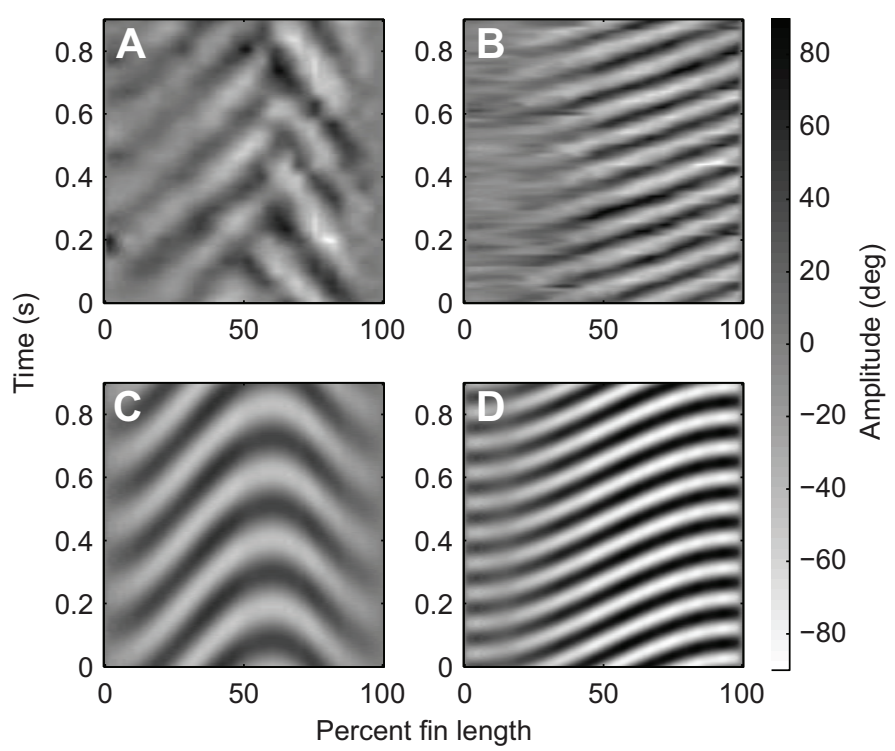

Fig. 10. Traveling wave amplitude comparison across behaviors for a set of representative trials. Grayscale indicates amplitude of fin movement in degrees. (A) Hovering experimental data. (B) Surging experimental data, swimming velocity of $19.7 \mathrm{~cm} \mathrm{~s}^{-1}$. (C) Hovering simulated data. (D) Surging simulated data.

This error can be found in Fig. 9B, as well as in the sentence 'The amount of stretch might not be insignificant: as Fig. 9B shows, the edge of the fin is 1.3 times the length of the fin base for a hovering fish.' This sentence should read 'The amount of stretch might not be insignificant: as Fig. 9B shows, the edge of the fin is 1.2 times the length of the fin base for a hovering fish.'

The corrected figures appear here.

The authors apologise for any inconvenience, and assure readers that these errors do not affect any other part of the analysis or conclusions. 


\title{
Kinematics of the ribbon fin in hovering and swimming of the electric ghost knifefish
}

\author{
Ricardo Ruiz-Torres ${ }^{1}$, Oscar M. Curet ${ }^{2, *}$, George V. Lauder ${ }^{3}$ and Malcolm A. Maclver ${ }^{1,2,4, \dagger}$ \\ ${ }^{1}$ Northwestern University Interdepartmental Neuroscience Program, Northwestern University, Chicago, IL, USA, \\ ${ }^{2}$ Department of Mechanical Engineering, Northwestern University, Evanston, IL, USA, ${ }^{3}$ Department of Organismic and \\ Evolutionary Biology, Harvard University, Cambridge, MA, USA and ${ }^{4}$ Department of Biomedical Engineering, \\ Department of Neurobiology, Northwestern University, Evanston, IL, USA \\ *Present address: Department of Ocean and Mechanical Engineering, Florida Atlantic University, Boca Raton, FL, USA \\ ${ }^{\dagger}$ Author for correspondence (maciver@northwestern.edu)
}

\begin{abstract}
SUMMARY
Weakly electric knifefish are exceptionally maneuverable swimmers. In prior work, we have shown that they are able to move their entire body omnidirectionally so that they can rapidly reach prey up to several centimeters away. Consequently, in addition to being a focus of efforts to understand the neural basis of sensory signal processing in vertebrates, knifefish are increasingly the subject of biomechanical analysis to understand the coupling of signal acquisition and biomechanics. Here, we focus on a key subset of the knifefish's omnidirectional mechanical abilities: hovering in place, and swimming forward at variable speed. Using high-speed video and a markerless motion capture system to capture fin position, we show that hovering is achieved by generating two traveling waves, one from the caudal edge of the fin and one from the rostral edge, moving toward each other. These two traveling waves overlap at a nodal point near the center of the fin, cancelling fore-aft propulsion. During forward swimming at low velocities, the caudal region of the fin continues to have counter-propagating waves, directly retarding forward movement. The gait transition from hovering to forward swimming is accompanied by a shift in the nodal point toward the caudal end of the fin. While frequency varies significantly to increase speed at low velocities, beyond approximately one body length per second, the frequency stays near $10 \mathrm{~Hz}$, and amplitude modulation becomes more prominent. A coupled central pattern generator model is able to reproduce qualitative features of fin motion and suggest hypotheses regarding the fin's neural control.
\end{abstract}

Supplementary material available online at http://jeb.biologists.org/cgi/content/full/216/5/823/DC1

Key words: aquatic locomotion, gymnotiform swimming, swimming gait transition.

Received 25 June 2012; Accepted 5 November 2012

\section{INTRODUCTION}

Locomotion using undulating dorsal or anal fins is a mode of fish swimming that has evolved independently in a number of unrelated teleost fish clades. Fishes swimming in this manner include the 200 species of South American weakly electric knifefish (Gymnotidae) (Albert and Crampton, 2005), approximately 10 species of African and Asian knifefish (Notopteridae), the African aba aba (Gymnarchus niloticus), the bowfin (Amia calva), and several deepsea fishes such as the oarfish (Regalecus glesne). Undulation of an elongated fin to produce thrust (Fig. 1) appears to confer several unusual capabilities, including the ability to swim with minimal or no body bending, reversal of swimming direction in a fraction of a second, and movement along the dorsoventral axis (Blake, 1983; Nanjappa et al., 2000; Lannoo and Lannoo, 1993; MacIver et al., 2001; Curet et al., 2011a).

South American weakly electric knifefish are a common model system for studying the neurobiology of sensory processing in vertebrates (for reviews, see Turner et al., 1999; Fortune, 2006) [for an understanding of the biomechanics and the neural control of movement, see Shirgaonkar and others (Shirgaonkar et al., 2008; MacIver et al., 2010; Cowan and Fortune, 2007); and for inspiring novel propulsion and sensing techniques for high agility underwater robots, see Epstein and others (Epstein et al., 2005; Epstein et al., 2006; MacIver et al., 2004; Curet et al., 2011a; Solberg et al., 2008;
Boyer et al., 2012)]. The subject of the present study is one species of knifefish, the weakly electric black ghost knifefish, Apteronotus albifrons (Linnaeus 1766). Like other knifefish, it propels itself by sending traveling waves along an extended anal fin attached to the ventral midline of the fish (Fig. 1A,B). The forward extension of the leading edge of this fin has moved the anus to position where it is on a vertical with the eye (Albert and Crampton, 2005), a testament to the selection pressures that underlie this unusual body plan.

Blake (Blake, 1983) studied the fin kinematics of several knifefish and concluded that ribbon-fin locomotion probably arose as a specialization for high maneuverability. Blake presented results on the characteristics of the traveling wave along the fins of knifefish during forward motion, such as maximum amplitude, wavelength and traveling wave frequency. The present study examines these characteristics with the use of high-speed video and two-dimensional markerless motion capture of the bottom edge of the fin, which allows for a detailed temporal and spatial analysis of fin waveforms.

Earlier work by our group has quantified the high level of agility of the black ghost knifefish. We have shown that the space in which the fish can sense prey extends out in all directions approximately one-third of a body length (Snyder et al., 2007). As with the highly maneuverable echolocating bat, the black ghost travels the distance of its sensory range in approximately one sensorimotor delay time 
at typical hunting velocities (Nelson and MacIver, 2006; Snyder et al., 2007). In order for prey to stay in sensory range, therefore, the fish needs to execute maneuvers to reach all points of its sensorium in a short amount of time with little if any increase in distance from prey to the sensory surface following detection. Consistent with such tight coupling between the sensory and mechanical dimensions of the fish, in prior work we have shown that the volume of water in which the fish can reach the prey in the time span of one to two sensorimotor delay times is a close approximation to its omnidirectional sensorium (Snyder et al., 2007). This maneuverability occurs despite holding the body axis semi-rigidly straight. Besides being a useful system for understanding the fundamental mechanical principles of multi-directional agility, high maneuverability with relative axial rigidity forms a compelling basis for inspiring new designs for underwater vehicles (MacIver et al., 2004; Curet et al., 2011b).

The anal fin of ghost knifefish is of particular interest as a locomotor structure because of the novel kinematic patterns described here. In contrast to the kinematics observed in previous studies of undulatory locomotion in fishes (e.g. Jayne and Lauder, 1995), in which a single wave of bending passes from head to tail down the body and changes in speed are mostly driven by alteration of bending frequency, in ghost knifefish there are dual traveling waves with a nodal point (Curet et al., 2009; Curet et al., 2011a), as also reported in preliminary findings for the knifefish Eigenmannia (Sefati et al. 2010; Sefati et al. 2012). As for Eigenmannia, these two fin waves travel in opposite directions to the nodal point, and the location of this point changes as speed changes, suggesting that the generation of counter-propagating waves might be a general strategy across taxa.

The goals of this paper are to: (1) provide an analysis of basic kinematic features of ribbon-fin movement during hovering and forward swimming by describing the two fin waves and changes in the nodal point; (2) characterize kinematics of the gait transition from hovering to forward swimming; and (3) use a pre-existing model of coupled central pattern generators to generate fin kinematics similar to those measured for insight into the fin's neural control.

\section{MATERIALS AND METHODS}

All data, analysis code, coupled CPG model, and behavioral movies are available on a companion website (Ruiz-Torres et al., 2012). Movies combining the original behavioral movies with animations of the corresponding digitized fin edge are available as supplementary material Movies 1-6.

Several terms are used throughout the remainder of the study (Fig. 1C). We will call the number of spatial periods of the sinusoidal waveforms typically along the fin the 'number of undulations'. If we idealize all undulations as having the same wavelength, this is simply the length of the fin divided by the wavelength, the reciprocal of specific wavelength (Blake, 1983; Shirgaonkar et al., 2008). Traveling waves originating from the anterior edge of the ribbon fin and proceeding toward the tail will be termed 'head waves', and those from the posterior edge and proceeding toward the head 'tail waves' (Sefati et al., 2012). The position along the fin where these two traveling waves overlap is termed the 'nodal point' (Sefati et al., 2012). Hovering refers to the stabilization of position, with nearzero movement of the fish's center of mass with respect to the external world. In this study, this occurs in still water within a 'refuge', typically a plastic cylinder or rectangular cuboid sized so that the fish fits within it with a few centimeters gap to all surfaces. When placed within a refuge, electric knifefish swim so as to maintain a fixed position with respect to the refuge, called refuge tracking behavior (Cowan and Fortune, 2007; Rose and Canfield, 1993). Refuge tracking is a significant convenience for the positioning of video cameras. Forward swimming is called 'surging'. In this study, this occurs in moving water within a flow tunnel as the fish holds station (typically within a refuge) pointing upstream.

\section{Fish}

Two adult black ghost knifefish were used. One $12.0 \mathrm{~cm}$ long fish, with an anal fin measuring $9.5 \mathrm{~cm}$ in length at its base (along its insertion to the body), was used for observing hovering, and one $14.2 \mathrm{~cm}$ long fish with an anal fin $10.5 \mathrm{~cm}$ long at its base was used for observing surging. The fin length was used as a normalizing constant for the analysis of the number of undulations along the fin, computed as fin base length divided by the mean wavelength of the undulations comprising the traveling wave (Fig. 1C). Fish were kept in a light-tight enclosure that was maintained on a $12 \mathrm{~h}: 12 \mathrm{~h}$ light:dark photoperiod. Water temperature in the tanks was kept at $28 \pm 1.0^{\circ} \mathrm{C}$ and the $\mathrm{pH}$ at $7.0 \pm 0.1$. They were fed live or frozen blackworms on a daily basis.

This study was conducted within the guidelines outlined by the USA National Research Council's 'Guide for the Care and Use of Laboratory Animals', and all protocols were approved by the Institutional Animal Care and Use Committees at Northwestern University and Harvard University.

\section{Behavior and video acquisition}

Two different swimming behaviors were studied, hovering and surging, as defined in the Introduction. During data collection for hovering behavior, the movement of the fin was observed while the fish was in a $50 \times 25 \times 28 \mathrm{~cm}$ tank of still water. During data collection for surging behavior, movement of the fin was observed while the fish was holding station in a variable speed flow tunnel with a working area of $28 \times 28 \times 80 \mathrm{~cm}$. The flow velocity was varied between 2.6 and $32.6 \mathrm{~cm} \mathrm{~s}^{-1}$. In all but one trial (surging at $14.9 \mathrm{~cm} \mathrm{~s}^{-1}$ ), the fish held station with respect to a transparent Plexiglas refuge. We used a $15.0 \times 5.0 \times 6.3 \mathrm{~cm}$ refuge $($ length $\times$ width $\times$ depth) for recording hovering, and a $15.0 \times 3.8 \times 5.2 \mathrm{~cm}$ refuge for recording surging. Refuges were open at both ends.

Video was recorded using a camera placed under the water tank pointing upwards (Fig. 1B). One sequence of hovering was recorded with a high-speed video camera (EX-F1, Casio, Tokyo, Japan) at 300 frames s$^{-1}$ and $512 \times 384$ pixel resolution and was 530 frames in duration. Five sequences of surging were recorded with a different high speed video camera (FASTCAM 1024PCI, Photron USA, San Diego, CA, USA) at 1000 frames s $^{-1}$ and $1024 \times 1024$ pixel resolution. Each sequence was 1000 frames in duration.

\section{Fin edge digitization}

The ribbon fin edge was digitized using a semi-automated process using custom software coded in MATLAB (The MathWorks, Natick, MA, USA). The digitizing was performed by manually selecting points on the fin edge and fitting a cubic spline to them. The number of manually selected points varied from frame to frame according to the complexity of the waveforms along the fin edge, ensuring that the resulting spline did not visibly depart from the outline of the fin edge. The fitting of points to the fin edge shape occurred every two to five frames. The movement of the fin edge in between these two to five frames was interpolated from the two spline-fitted fin waveforms and later visually inspected to verify congruence with image data. If the digitized fin edge visibly departed from the imaged fin edge within the interpolated frames, then an intermediate frame was selected for digitization or additional spline 

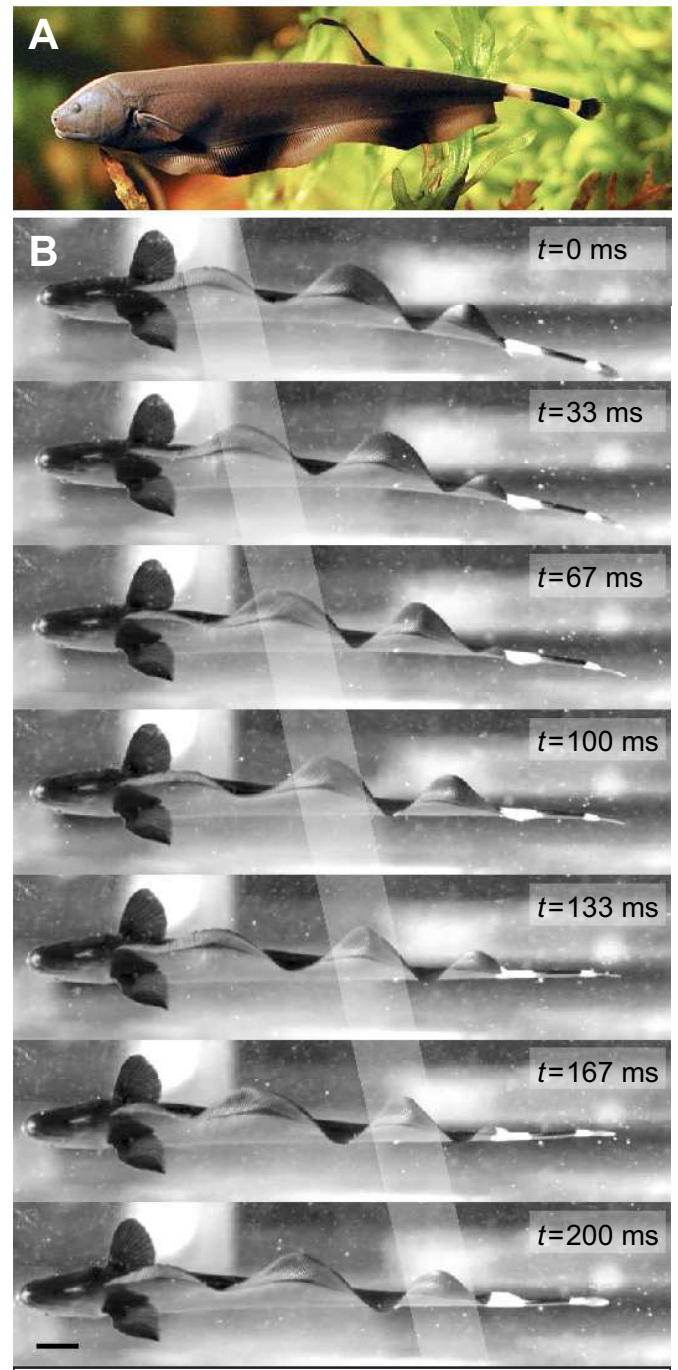

C

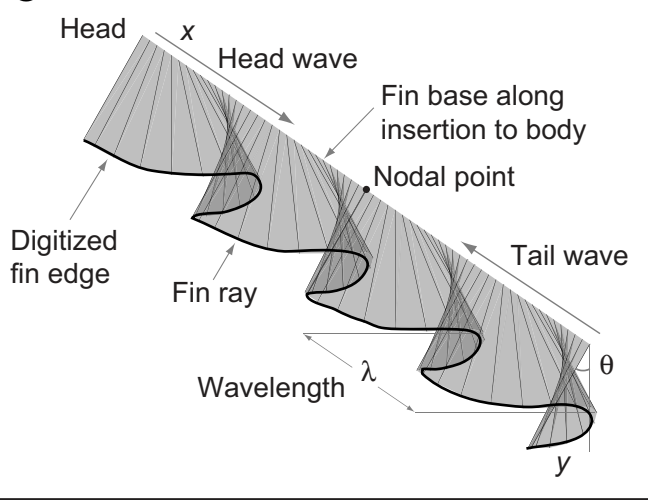

Fig. 1. The black ghost knifefish, Apteronotus albifrons. (A) Photograph courtesy of Per Erik Sviland. (B) Ribbon fin generating head waves as the fish holds its position against a headfirst flow. The lighter band follows a traveling wave peak as it travels towards the tail. Scale bar, $1 \mathrm{~cm}$. Flow velocity, $14.9 \mathrm{~cm} \mathrm{~s}^{-1}$, from left to right. (C) Definition of some terms used in the paper. The number of undulations is the number of complete spatial periods (one of four illustrated by $\lambda$ ); head waves are the traveling waves moving from head to tail; tail waves are the traveling waves moving from tail to head; $\theta$ is the angle from the midsagittal plane; nodal point is where head and tail waves overlap; fin ray denotes borders of discrete segments of the fin $1 / 100$ th of the fin base length. Digitized fin edge and fin base are also shown. points were added as needed. The body centerline was digitized using a similar approach.

The anal fin extends from near the insertion of the pectoral fins to close to the vestigial caudal fin. It is black throughout but for the caudal $\sim 1 \mathrm{~cm}$ segment, where it becomes transparent (visible in Fig. 1A, where the dark ventral fin becomes semi-transparent, terminating just caudal to the anterior white band on the tail). The fin edge profile for the transparent segment was not digitized as it could not be reliably seen. This segment, however, is where the fin tapers to a thin edge and is likely to be less significant in terms of thrust production. All calculations involving fin length used the fin base insertion length measured directly from the specimens rather than the edge length derived from digitized video (as fin edge length varies with the amplitude of the waveforms present along the fin).

The fin edge position data was then processed frame by frame in such a way that both the tail and head ends would be fixed at predetermined points, and the projection of the fin insertion on the image plane would conform to a straight line. To accomplish this, the angle of the body was estimated using the rostral and caudal ends of the digitized centerline. The centerline of the body and the fin edge data were rotated by that value in the opposite direction so that the head and tail were on the horizontal axis. The digitized centerline was then subtracted from the fin coordinates. Thus, if the body and therefore the centerline were completely straight, the fin coordinates would not be changed; if the centerline bowed away from the horizontal axis by a certain amount of $y$-offset, this offset would be subtracted from the corresponding fin coordinate. Last, the fin was translated so that the anterior end was at the origin and the length along the body axis was normalized. A cubic spline was fit to the modified fin data.

For further processing it was desirable that the distance between points along the lengthwise direction was identical. To accomplish this, the data were resampled at fixed intervals along this axis. Two different spatial sampling rates were used: one that split the fin into 100 segments, and one that split the fin into 1000 segments. For the 100 segment sampling, we refer to the segments as 'rays', their number being similar to the actual number of rays (lepidotrichia) that together with the fin membrane make up the anal fin (Albert, 2001). In one movie, where the fish's body was rolled by $\sim 60 \mathrm{deg}$ (estimated visually), an additional correction was performed, where the estimated angle of the fin with respect to the midsagittal plane of the fish body ( $\theta$ in Fig. 1C) was divided by the cosine of the roll angle in order to obtain a more accurate projection. We estimated the correct angle as:

$$
\theta(x)=\sin ^{-1}\left[y(x) / h_{\mathrm{fin}}(x)\right],
$$

where $\theta(x)$ is the angle, $y(x)$ is the measured fin displacement from the midsagittal plane and $h_{\text {fin }}(x)$ is the height of the fin - as measured from an anesthetized animal - at distance $x$ (Fig. 1C) from the rostral end of the fin towards the caudal end along the line of insertion of the fin into the body.

Fin wave amplitudes are presented as angular displacement from the midsagittal plane in degrees. Position along the fin is presented as a percentage of its edge length from the rostral end $(0 \%)$ to the caudal end $(100 \%)$. The position of the nodal point was estimated by inspection from the wave amplitude data presented in the left column of Fig. 2, where it is shown as a red line.

The envelope of the undulations traveling across the fin was found by averaging the maximum displacement of each ray tip (defined above as a spatial location each 1/100th of fin length) during each trial.

The length of the digitized fin edge $\left(S_{\text {fin }}\right)$ was calculated at each frame and averaged over every trial. One centimeter was added to 

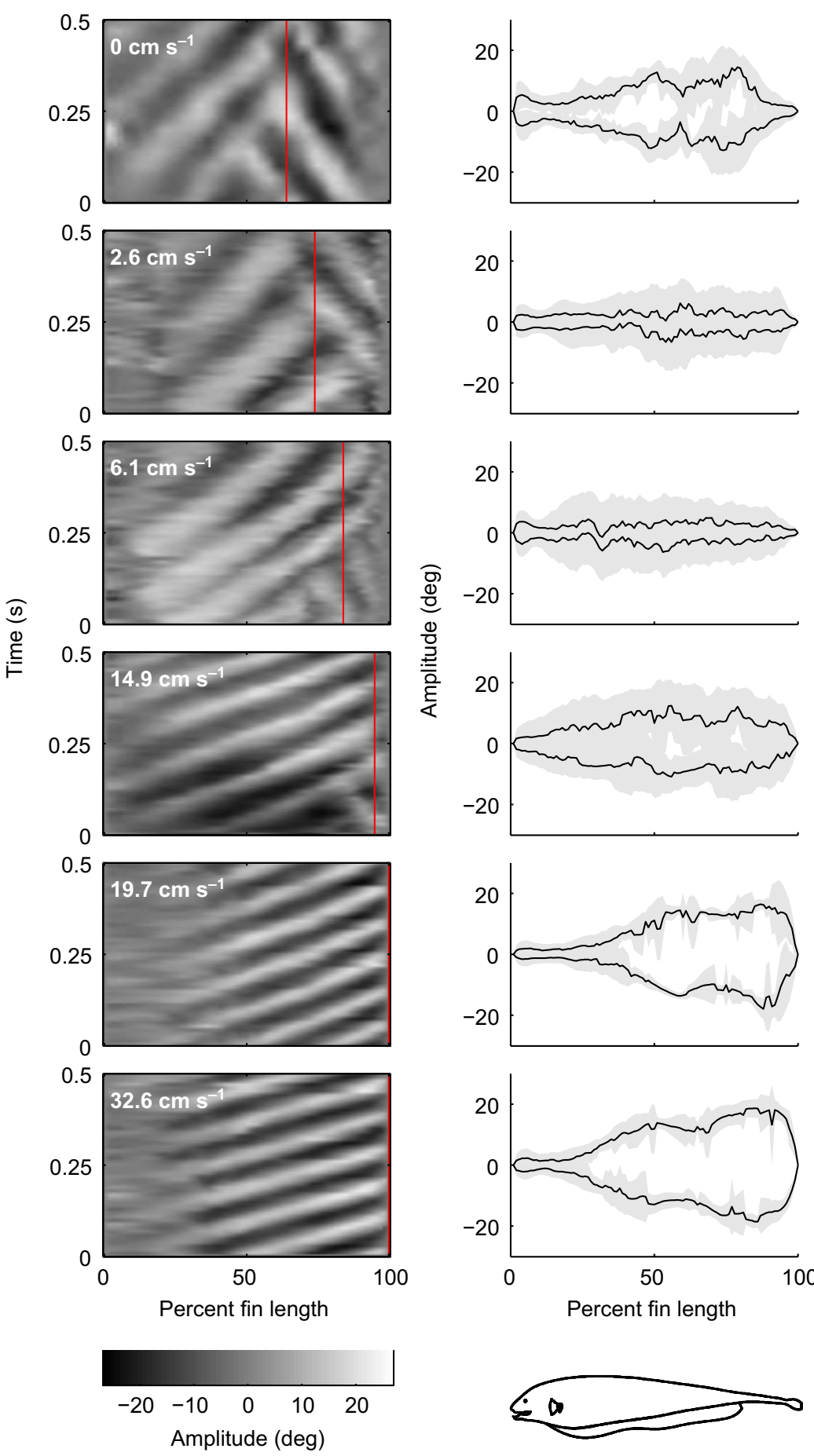
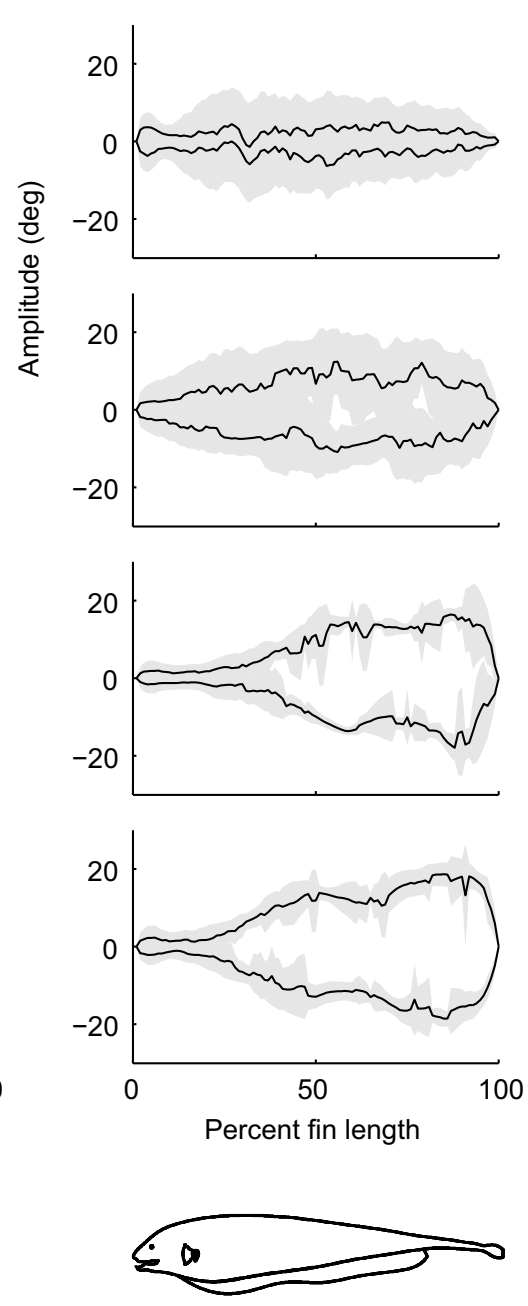

Fig. 2. Comparison of traveling wave amplitude patterns and wave envelope for different swimming velocities. Two counterpropagating waves are seen at low swimming velocities. The fraction of the fin occupied by the tail wave becomes shorter as the swimming velocity increases. Grayscale map is for amplitude in degrees. Estimated nodal point position for each swimming velocity is shown as a vertical red line. The first $500 \mathrm{~ms}$ of data are shown per trial for comparison. Trial duration varies amongst data sets. Mean wave envelope is shown in the right column. Gray area ranges vertically by \pm 1 s.d. of the peak magnitude of fin angle across the trial $(N=530$ frames for $0 \mathrm{~cm} \mathrm{~s}^{-1}, N=1000$ for all other cases). the digitized lengths to account for the non-digitized transparent portion of the fin. The mean values for each trial were normalized by the measured fin base length $\left(L_{\text {fin }}\right)$. In order to indirectly estimate the momentum imparted to the fluid by the fin, the area under the curve of absolute fin angular displacement versus percent fin length was calculated by the rectangle method using the resampled angular displacement data. Then an average area during a trial can be calculated by summing the areas over the frames of a trial and dividing by the number of frames used. We calculated a normalized area for any given trial by dividing the average area of that trial by the average area of the trial with the maximum average area. Because segment length is the same for all of these curves, it drops out of the calculation of normalized area. Therefore, normalized area was calculated by summing all angular displacements for a given trial, dividing by the number of frames and then dividing by the same quantity for the trial in which the quantity was the maximum.

\section{Ray oscillation frequency}

A discrete fast Fourier transformation (FFT) was performed on the time series of angular fin displacements at each of the 100 fin rays of the coarsely sampled data to determine the frequency of oscillation of every ray throughout each trial. Signal power at each oscillating frequency was obtained by squaring the FFT amplitude at that point and was normalized by the maximum value for each trial. 


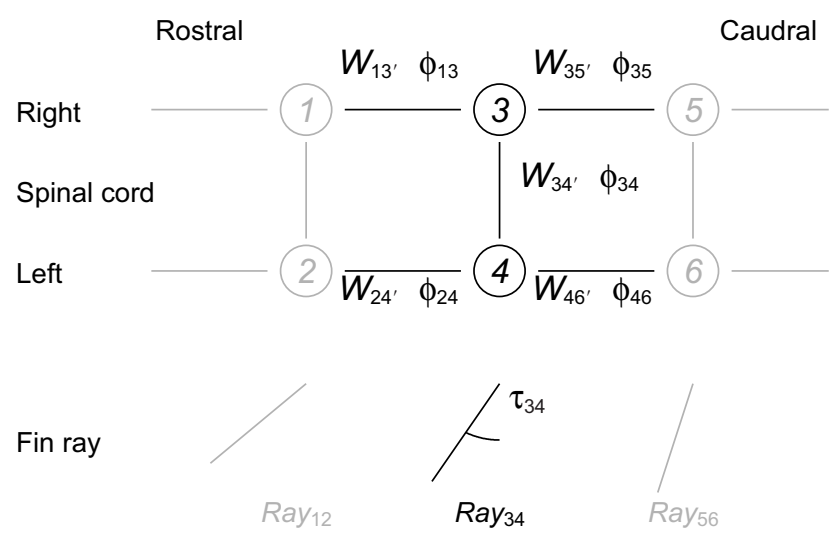

Fig. 3. Diagram of the central pattern generator model. The circles represent the oscillators, which are connected to their neighbors with weight $W$ and phase angle difference $\phi . \tau_{N}$ designates the amplitude for ray $N$.

\section{Number of undulations}

Every frame of the finely sampled fin data (1000 segments) was transformed into the spatial frequency domain by applying a $2^{14}$ point FFT. The signal power was calculated for each frame by squaring the FFT amplitude at each spatial frequency point and was then normalized by the maximum value for each trial. The number of undulations along the fin was obtained by finding the spatial frequency at which signal power was maximal at each frame and then averaging across frames.

\section{Traveling wave velocity}

Traveling wave velocity for each trial was calculated by summing the number of times each ray crossed the centerline, dividing by two to obtain the number of full cycles of the ray (as opposed to half cycles) and calculating the average across all rays. The result was then multiplied by the fin length and divided by product of the number of undulations along the fin and the duration of the trial. In the cases where head and tail waves were present, wave velocity was calculated in the same manner, but for the head waves only using rays anterior to the nodal point, and for the tail wave only with rays posterior to the nodal point.

\section{Neural control model}

A model of the neural control of the fin was developed based on the lamprey-inspired model described in Ijspeert et al. (Ijspeert et al., 2007). Two columns of phase oscillators make up a central pattern generator (CPG). Each oscillator pair controls a single ray, one activating the muscle that deflects the ray to the left of the fish body and the other to the right. A diagram showing the connections of a pair of oscillators is shown in Fig. 3.

Every oscillator is phase coupled with at most three other oscillators: the ones located directly rostral and caudal to it and the one across the midline from it. The two most caudal and two most rostral oscillators have only two connections each. The equation governing the motion of the rays is:

$$
\dot{\theta}_{i}=2 \pi v_{i}+\sum_{j} r_{j} W_{i j} \sin \left(\theta_{j}-\theta_{i}-\phi_{i j}\right),
$$

where $\theta_{i}$ and $\dot{\theta}_{i}$ represent the phase and rate of change of the phase of oscillator $i$, respectively, $v_{i}$ is its intrinsic oscillating frequency, $r_{j}$ is the amplitude of oscillator $j$ and $W_{i j}$ represents the coupling weights between the two oscillators. The coupling weights were
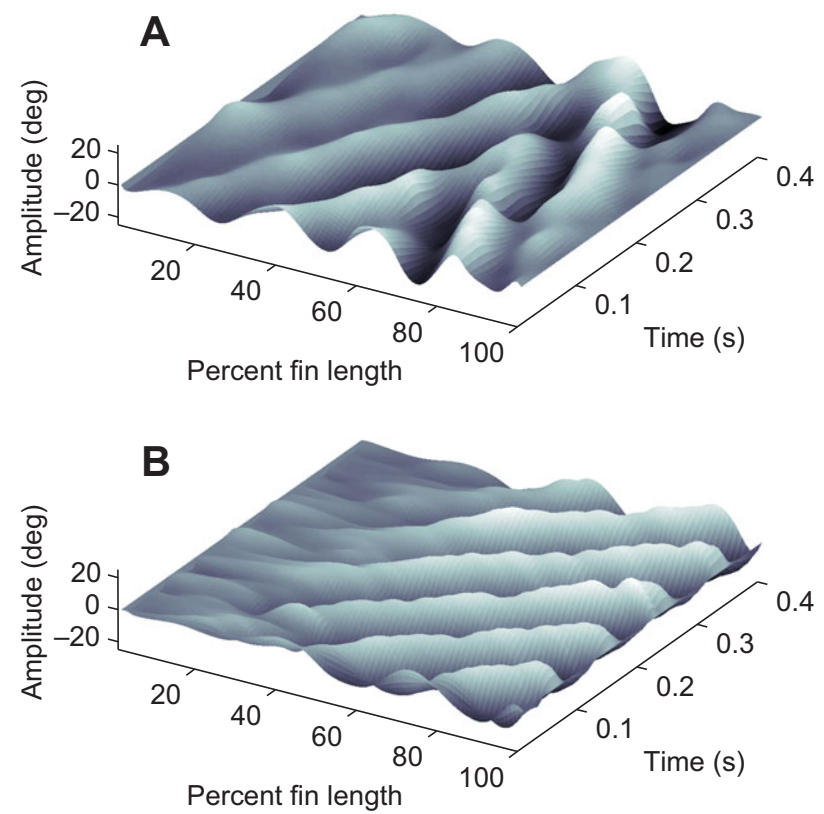

Fig. 4. Traveling wave comparison between (A) hovering and (B) surging at $19.7 \mathrm{~cm} \mathrm{~s}^{-1}$. A single wave travels caudally during forward swimming, while two waves traveling towards each other are observed while hovering. Grayscale is used to enhance amplitude information. The rostral end of the fin is at $0 \%$ on the fin length axis.

set to 200 for every pair of ipsilaterally and contralaterally adjacent oscillators and to 0 for all other pairs (e.g. with respect to Fig. 3, $W_{13}=W_{35}=W_{34}=200$, and $\left.W_{23}=W_{36}=0\right)$. The term $\phi_{i j}$ indicates the phase difference between the two connected oscillators to which the numerical solution should converge. For the oscillators connected along the rostro-caudal axis, the value is set based on the desired number of undulations that are to be present along the fin. For the pair of oscillators controlling each ray, the value of $\phi_{i j}$ was fixed to $\pi$ radians, causing their actions upon the ray to be antagonistic. For ipsilateral pairs of oscillators, the value of $\phi_{i j}$ was set to 0.016 radians. The values of $v_{i}$ and $r_{i}$ depend on the value of driving input $d_{i}$ as described in the following equations:

$$
\begin{aligned}
& v_{i}=C V_{1} d_{i}+C V_{2}, \\
& r_{i}=C R_{1} d_{i}+C R_{2},
\end{aligned}
$$

where $C V_{1}, C V_{2}, C R_{1}$ and $C R_{2}$ are constants with values of $3.7 \mathrm{~Hz}$, $0.3 \mathrm{~Hz}, 0.065$ radians and 0.196 radians, respectively. In the original model the value of $C V_{1}$ was set to 0.2 , creating a slow motion appropriate for the simulation of a salamander, whereas a higher value is necessary to more accurately simulate the quick motion of the knifefish fin. The rest of the parameters were taken from Ijspeert et al. (Ijspeert et al., 2007) and were found to be appropriate for our simulations. The change in oscillator phase is translated to ray motion by the following equation:

$$
\tau_{i, i+1}=\alpha_{i, i+1} r_{i, i+1}\left[\cos \left(\theta_{i}+1\right)-\cos \left(\theta_{i+1}+1\right)\right],
$$

where $\tau_{i, i+1}$ is the amplitude of the ray deflection, $\theta_{i}$ and $\theta_{i+1}$ are the output of the left and right oscillators for ray $i$ and $\alpha_{i, i+1}$ is a function that multiplies the amplitude of the ray to adjust the overall amplitude envelope. This function is positioned at the center of the fin with a value of 0 at both ends and 1 in the center. 


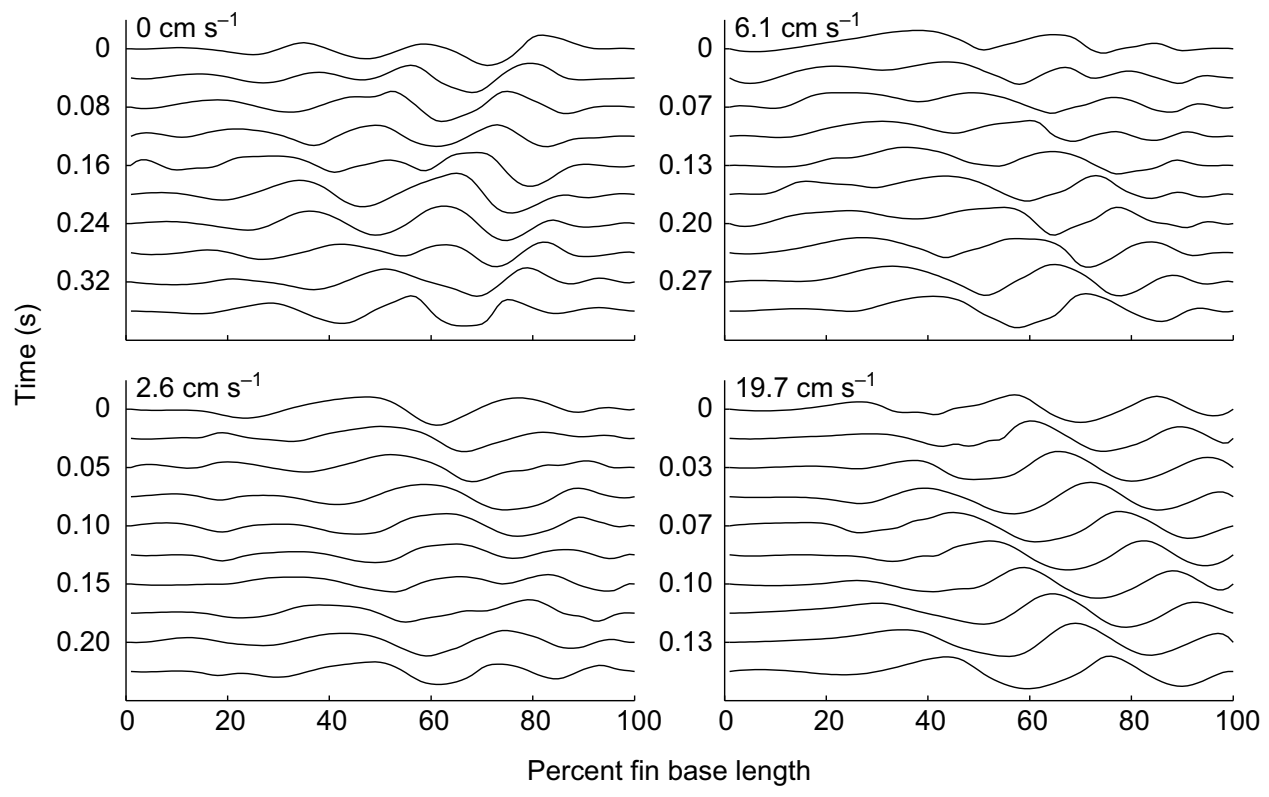

Fig. 5. Ten snapshots of digitized ventral fin edges over the full fin length for hovering and surging. The rostral end of the fin is at $0 \%$ on the $x$-axis. Note that the vertical time interval varies across the plots. The vertical spatial scale is fixed.

The Euler integration method with a time step of $10 \mathrm{~ms}$ was used to solve the equation. A graphical interface was programmed to allow for the adjustment of the driving input $(d)$ while the simulation ran. The driving input for the whole fin was set as a two-segment piecewise linear function. The equation for the driving force of one side of the fin is:

$$
d(x)=\left\{\begin{array}{cc}
\frac{\left(d_{\mathrm{b}}-d_{\mathrm{a}}\right) x}{x_{\mathrm{b}}-x_{\mathrm{a}}}+d_{\mathrm{a}}, & 1 \leq x \leq x_{\mathrm{b}} \\
\frac{\left(d_{\mathrm{c}}-d_{\mathrm{b}}\right) x}{\left(x_{\mathrm{c}}-x_{\mathrm{b}}\right)}+d_{\mathrm{b}}, & x_{\mathrm{b}}<x \leq x_{\mathrm{c}}
\end{array}\right\},
$$

where $x_{\mathrm{a}}$ and $d_{\mathrm{a}}$ are the position and driving input of the first ray, $d_{\mathrm{b}}$ is the input of the ray at the nodal point position $x_{\mathrm{b}}$ (where two traveling waves along the fin, if present, meet) and $d_{\mathrm{c}}$ is the input of the last ray, at location $x_{\mathrm{c}}$.

Controlling input to the rostral end $\left(d_{\mathrm{a}}\right)$, nodal point $\left(d_{\mathrm{b}}\right)$ and caudal end $\left(d_{\mathrm{c}}\right)$ of the fin differentially allows us to generate traveling waves similar to those found in the knifefish anal fin. When the drive signal is higher at the rostral end $\left(x_{\mathrm{a}}\right)$ than in other locations of the fin, waves start traveling in a caudal direction. The opposite is true when drive is higher at the caudal end $\left(x_{\mathrm{c}}\right)$. When the drive is lower at the nodal point $\left(x_{\mathrm{b}}\right)$ than at the ends of the fin, counterpropagating waves travel toward the nodal point.
In summary, wave direction is controlled by the direction of the input drive gradient, the number of undulations of the fin is proportional to the drive gradient, and individual ray frequency increases with the drive input to that particular ray. A nodal point occurs where two drive gradients converge. Wave amplitude is given by the envelope function $\alpha$. Table 2 shows the parameter values used for all simulations.

All statistical values are reported as means \pm s.d., unless indicated otherwise.

\section{RESULTS}

\section{Basic features of ribbon fin movement}

Fig. 4 shows the digitized fin edge for the first $0.4 \mathrm{~s}$ of the hover trial and the $19.7 \mathrm{~cm} \mathrm{~s}^{-1}$ surge trial, illustrating some of the basic differences between hover and surge. Fig. 5 shows 10 snapshots of the fin edge shape for these trials and several others in finer detail. Hovering is distinguished from surging by the nearly equal length of the fin carrying head waves and tail waves, canceling any surge force that might otherwise occur. When surging forward, most or all of the fin carries head waves.

\section{Hovering}

Hovering is characterized by more undulations along the fin than during surge (Fig 6, Fig. 7B, Table 1), lower frequency of fin oscillation (Fig.7A, Fig. 8, Table 1), higher wave amplitude

Table 1. Traveling wave parameters across all hover and surge trials

\begin{tabular}{|c|c|c|c|c|c|}
\hline Swim speed $\left(\mathrm{cm} \mathrm{s}^{-1}\right)$ & Frequency $(\mathrm{Hz})$ & No. undulations along fin & Wavelength $\left(\mathrm{cm}\right.$ wave ${ }^{-1}$ ) & Wave velocity $\left(\mathrm{cm} \mathrm{s}^{-1}\right)$ & Wave efficiency \\
\hline \multirow[t]{2}{*}{0} & $4.5 \pm 0.8$ & $3.8 \pm 0.7$ & $2.5 \pm 0.5$ & $11.4 \pm 3.0$ & $\mathrm{n} / \mathrm{a}$ \\
\hline & $3.9 \pm 4.5$ & $4.2 \pm 0.7$ & $2.3 \pm 0.4$ & $9.9 \pm 3.9$ & \\
\hline 2.6 & $5.1 \pm 1.5$ & $2.3 \pm 0.5$ & $4.5 \pm 1.0$ & $29.3 \pm 14.3$ & $\mathrm{n} / \mathrm{a}$ \\
\hline \multirow[t]{2}{*}{6.1} & $6.4 \pm 5.6$ & $2.7 \pm 0.7$ & $3.8 \pm 1.0$ & $21.6 \pm 11.3$ & $\mathrm{n} / \mathrm{a}$ \\
\hline & $8.3 \pm 8.9$ & $2.9 \pm 2.4$ & $3.6 \pm 3.0$ & $24.9 \pm 23.5$ & \\
\hline 14.9 & $9.3 \pm 1.2$ & $2.5 \pm 0.4$ & $4.2 \pm 0.7$ & $31.1 \pm 7.4$ & $\mathrm{n} / \mathrm{a}$ \\
\hline 32.6 & $9.9 \pm 2.8$ & $2.4 \pm 0.1$ & $4.4 \pm 0.2$ & $46.1 \pm 6.7$ & $0.7 \pm 0.1$ \\
\hline
\end{tabular}

At velocities of $0-14.9 \mathrm{~cm} \mathrm{~s}^{-1}$, characteristics of head waves are shown in the first row, and those of tail waves are shown in the second row. At velocities of 19.7 and $32.6 \mathrm{~cm} \mathrm{~s}^{-1}$, only head waves were present. Values are means \pm s.d. ( $N=530$ for $0 \mathrm{~cm} \mathrm{~s}^{-1}, N=1000$ for all other velocities). 


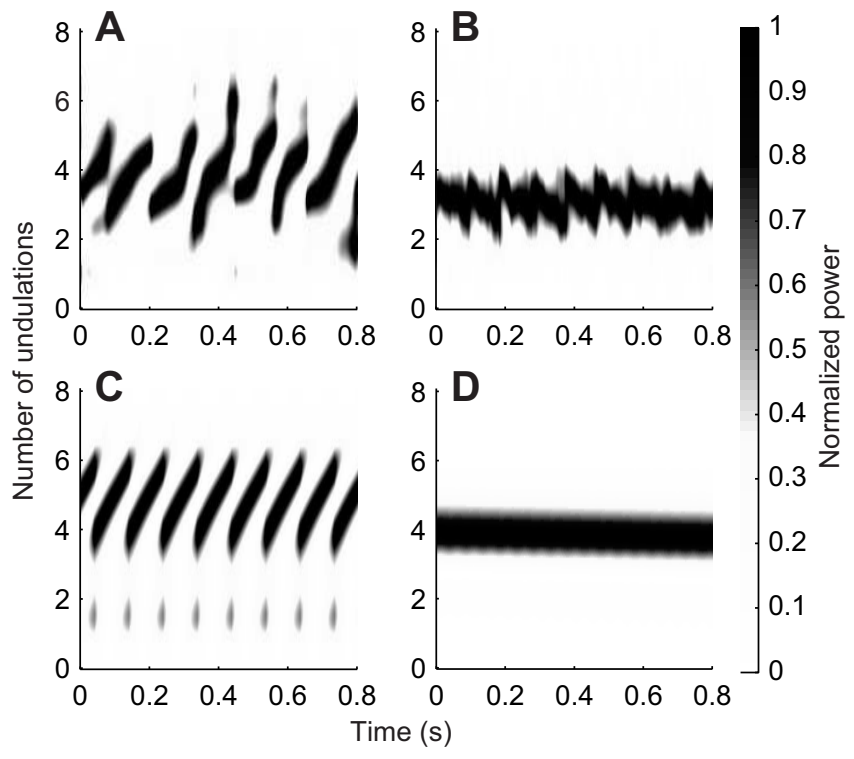

Fig. 6. Comparison of the number of undulations along the fin across behaviors. Wave power normalized by the maximum value is shown for each trial. (A) Hovering experimental data. (B) Surging experimental data $\left(19.7 \mathrm{~cm} \mathrm{~s}^{-1}\right)$. (C) Hovering simulated data. (D) Surging simulated data. Only the first $800 \mathrm{~ms}$ of data are shown per trial for comparison purposes. The total duration varies across trials.

compared with amplitudes at low surge velocities (Fig. 9C, Fig. 2) and a nodal point that is close to the middle of the fin (Fig.9D, Fig. 10), with slightly more fin length devoted to head waves than tail waves.

The sawtooth pattern in number of undulations shown in Fig. 6A is a result of inward head and tail waves overlapping at the nodal point. As two wave crests approach each other the total number of undulations on the fin increases, and when they overlap it decreases sharply. A plot showing this effect in a simulated data set is shown in Fig. 11.

\section{Hover to surge gait transition}

Fin amplitudes when swimming at different velocities, from hovering to surging at $32.6 \mathrm{~cm} \mathrm{~s}^{-1}$, are shown in Fig. 2. In still water, counter-propagating waves overlap at the nodal point close to the center of the fin. At $2.6 \mathrm{~cm} \mathrm{~s}^{-1}$, the lowest velocity tested, the nodal point shifts caudally (Fig. 9D), wave amplitude reduces by over a factor of two (Fig. 9C), the frequency of fin oscillation increases (Fig. 7A) and the traveling wave velocity increases (Fig. 7C). While the frequency of oscillation of tail waves is $0.6 \mathrm{~Hz}$ lower than head waves during hovering, the frequency of tail waves is $2.5 \mathrm{~Hz}$ higher when surging at $2.6 \mathrm{~cm} \mathrm{~s}^{-1}$ (Fig. 7A) and continues to be higher than the head wave at $6.1 \mathrm{~cm} \mathrm{~s}^{-1}$.

\section{Surging}

As swimming velocity increases, there are changes across many fin kinematic parameters. The least varying feature is the number of undulations along the fin, $2.6 \pm 0.2$ (Fig. 7B). From 2.6 to $14.9 \mathrm{~cm} \mathrm{~s}^{-1}$, the frequency of fin oscillation rose from $5.1 \mathrm{~Hz}$ at $2.6 \mathrm{~cm} \mathrm{~s}^{-1}$ to $9.3 \mathrm{~Hz}$ at $14.9 \mathrm{~cm} \mathrm{~s}^{-1}$ (values quoted for head waves only). The nodal point moves from near the middle of the fin to close to the posterior tip, while amplitude and wave envelope increase. The tail waves present on the fin at up to $14.9 \mathrm{~cm} \mathrm{~s}^{-1}$ do not appear to be significantly different from head waves (Fig. 7A-C, Table 1).
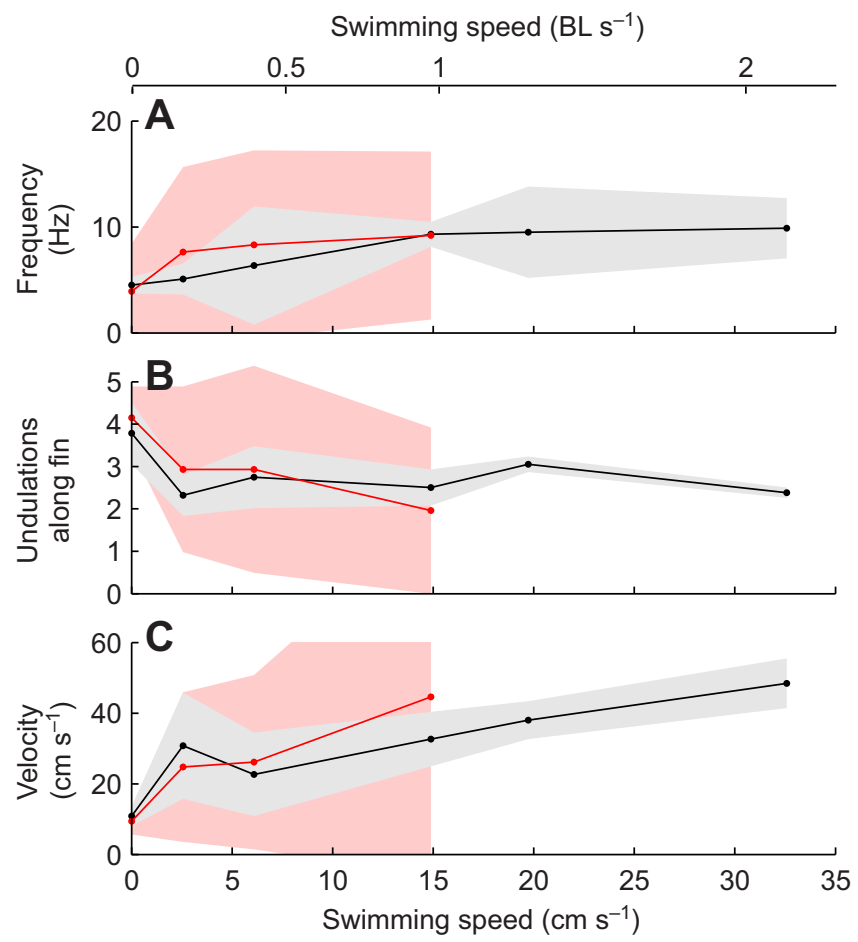

Fig. 7. Wave velocity characteristics for different swimming speeds. The red line is the tail wave, and the black line is the head wave. (A) Ray frequency; (B) number of undulations; (C) traveling wave velocity. Gray (pink) shaded area ranges vertically by \pm 1 s.d. $\left(N=530\right.$ frames for $0 \mathrm{~cm} \mathrm{~s}^{-1}$, $N=1000$ for all other cases).

From $14.9 \mathrm{~cm} \mathrm{~s}^{-1}$ through to the highest speed we tested $\left(32.6 \mathrm{~cm} \mathrm{~s}^{-1}\right)$, the change in frequency was small $(9.3$ to $9.9 \mathrm{~Hz})$, but two other aspects of the traveling waves change significantly: first, the counter-propagating tail wave disappears, and second, the amplitude of the head wave increases. In this high-speed regime, velocity increase appears to be through a slight increase in wave amplitude, a lengthening of the large amplitude region of fin movement visible in the wave envelope (Fig. 2) and an increase in wave velocity (mediated by a decrease in the number of undulations along the fin from 3.1 to 2.4, Fig. 7B). One other notable feature of the fin dynamics at these two velocities is that the wave envelope becomes highly tapered at the rostral end, flaring out in the caudal region of the fin (Fig. 2).

\section{Model}

The kinematics found in the experimental data were largely replicated with the CPG model. A comparison of the waves generated during both experimental and computer simulated trials can be seen in Fig. 6 (number of undulations), Fig. 8 (frequency) and Fig. 10 (amplitude). One discrepancy was that in the experimental data shown in Fig. 10, the position of the nodal point varies somewhat over the trial, from 51 to $63 \%$ of the fin length, while the nodal point did not vary its position in the simulation. This most likely arises because in the experimental situation, the nodal point will need to be constantly shifted by a small amount for the fish to hold station due to the complex fluid dynamics that are occurring. In the model these dynamics are absent.

A second discrepancy is that in the experimental data for surging, the traveling waves are moving at a constant rate along the ribbon fin, while in the computational results this rate is slightly less at the 


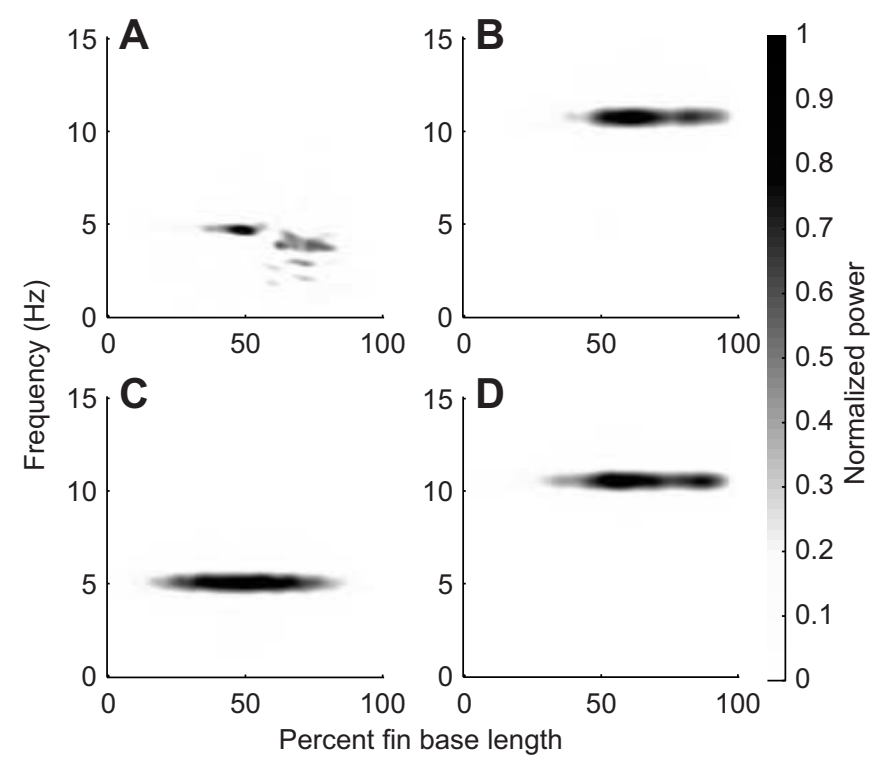

Fig. 8. Traveling wave frequency power analysis comparison across behaviors. (A) Hovering experimental data. (B) Surging experimental data, swimming velocity of $19.7 \mathrm{~cm} \mathrm{~s}^{-1}$. (C) Hovering simulated data. (D) Surging simulated data. Power is normalized by the maximum value for each trial. Note that the power band shown in B seems higher than the average frequency in Fig. 7A at $19.7 \mathrm{~cm} \mathrm{~s}^{-1}$. That is because in Fig. 7 frequency is averaged across all rays, including those with normalized power below the visible threshold in this figure.

anterior and posterior edges of the fin. This results from the fact that the oscillators at either end of the fin have fewer connections to other oscillators. The oscillators at the rostral and caudal ends of the fin are connected to two other oscillators: the rostral-most oscillator is connected to the oscillator immediately caudal to it and one contralateral oscillator, and the caudal-most oscillator is connected to the oscillator immediately rostral to it and one contralateral oscillator. All other oscillators are connected to three other oscillators: one rostral, one caudal and one contralateral. Because the drive to an oscillator increases with the number of oscillators connected to it, the terminal oscillators receive a smaller driving signal, which is translated into a slower movement. Presumably this is not seen in the experimental data because the weights of the connections from one neural oscillator to the next do not have to be the same for all of them, as they are in our model.

Surging data for the experimental (Fig. 8B) and simulated cases (Fig. 8D) show similarities in the frequency of highest power. The similarity in the location of highest power along the fin arises because we used the amplitude of the experimental wave envelope as the $\alpha$ term in Eqn 5 for the simulation. Because variations in wave amplitude lead to correspondingly larger contributions to the power spectrum for a given location along the fin, the result is that simulation and data are necessarily similar.

\section{DISCUSSION}

\section{Kinematics and swimming speed control}

There are multiple fin parameters that the fish can vary to precisely control its swimming speed: frequency, ray amplitude and number of undulations, for both head and tail traveling waves. In addition, it can control the position of the nodal point where these two counterpropagating waves meet along the fin. Our results show that the fish is controlling all of these parameters for the range of swimming speeds that we examined, $0-2.3$ body lengths per second. Below is
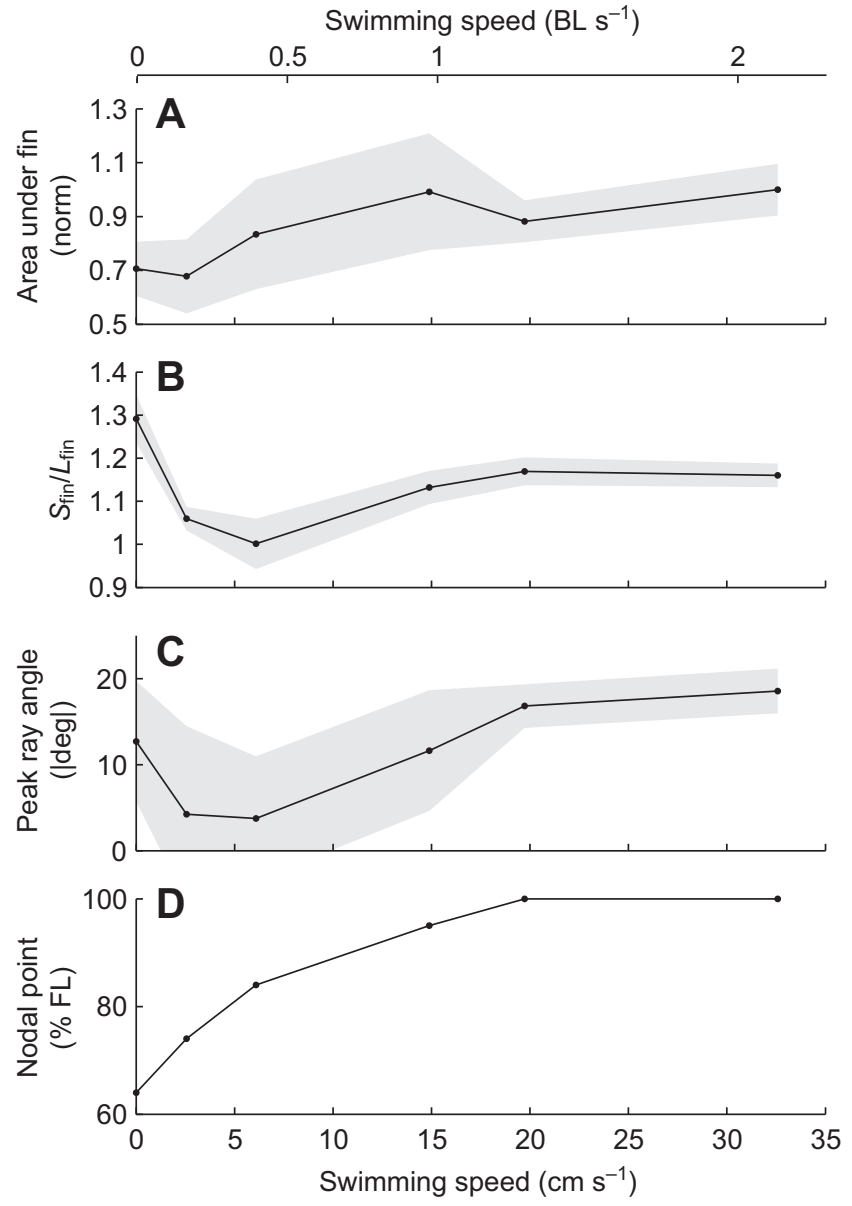

Fig. 9. Traveling wave variables as a function of swimming speed: (A) area under the fin, $(B)$ normalized fin edge length, $(C)$ peak ray angle and (D) nodal point location. The area under the fin is normalized with respect to the largest value. Fin edge length $\left(S_{\mathrm{fin}}\right)$ is normalized with respect to the fin base length $\left(L_{\text {fin }}\right)$. Gray area ranges vertically by \pm 1 s.d. No s.d. is shown for nodal point location as this was obtained through visual inspection of the amplitude profiles (see Materials and methods). FL, fin length. $N=530$ frames for $0 \mathrm{~cm} \mathrm{~s}^{-1}, \mathrm{~N}=1000$ for all other cases.

a summary of the control strategies used by the fish to reach and sustain those swimming velocities.

Hovering is characterized by the presence of two counterpropagating traveling waves with similar frequencies and numbers of undulations, with a nodal point slightly caudal to the midpoint of the fin. As swimming speed increases up to approximately one body length per second, the nodal point is moved towards the tail at a fast rate, and the number of undulations drops. In addition, the frequency of the counter-propagating waves increases, accompanied first by a substantial decrease in the ray amplitude and subsequently by an increase in the amplitude.

It is unclear why the fish would use counter-propagating waves for forward swimming as it is not energetically beneficial. One possibility is that the fish may need additional heave force gained from counter-propagating waves to counterbalance its weight because the fish is slightly negatively buoyant (Curet et al., 2011a). Another possibility is that counter-propagating waves along the fin confers stability and controllability advantages (Sefati et al., 2012). Counter-propagating waves allow positive or negative surge around zero to occur with smooth and linear changes in the nodal point position, rather than relatively large changes such as reversing the 


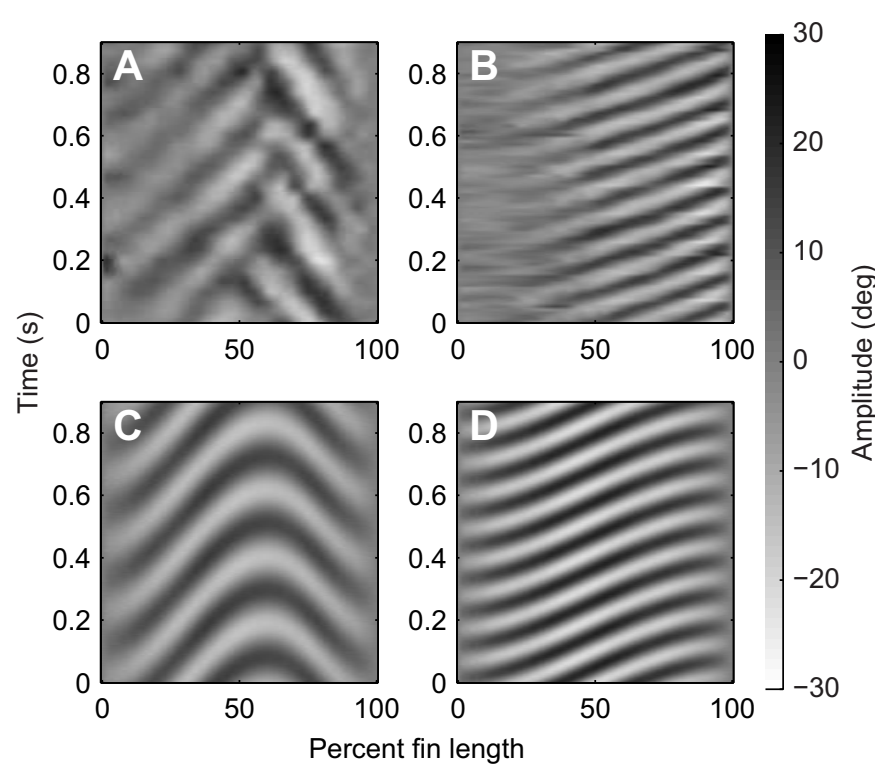

Fig. 10. Traveling wave amplitude comparison across behaviors for a set of representative trials. Grayscale indicates amplitude of fin movement in degrees. (A) Hovering experimental data. (B) Surging experimental data, swimming velocity of $19.7 \mathrm{~cm} \mathrm{~s}^{-1}$. (C) Hovering simulated data. (D) Surging simulated data.

direction of a single traveling wave along the fin (Sefati et al., 2012). Another advantage of counter-propagating waves is that they enhance stabilization against fore-aft perturbations in position, as the relative velocity between fin and water is increased for the traveling wave creating thrust against the perturbation, and decreased for the traveling wave producing thrust accentuating the perturbation. All of these advantages are true for both inward and outward counterpropagating waves. However, unlike inward counter-propagating waves, outward waves have never been observed along the fish fin. One possible reason is that outward waves reduce the size of the heave force and lead to larger variations in the force around the mean, something we have previously shown with a robotic knifefish fin (Curet et al., 2011a).

As swimming speed increases beyond approximately one body length per second, the fish seems to be precisely controlling both wave velocity and the amplitude of fin ray oscillation. In this range of velocities only head waves are present, and the traveling wave velocity increases monotonically as the swimming speed increases. However, the wave velocity is not exclusively controlled by frequency or number of undulations, but by a combination of both. (Wave speed is the frequency times the wavelength, in the idealized case of all undulations having the same length.) The fish reaches 1.4 body lengths per second by increasing the frequency and the ray amplitude. In addition, the fish changes the mean wave envelope of the rays in the caudal region of the fin, and decreases the amplitude of the rays in the rostral region of the fin. To reach the highest swimming velocity ( 2.3 body lengths per second), the fish increases the amplitude of the rays, the wave velocity (through a substantial decrease in the number of undulations) and the oscillation frequency. The wave envelope is similar to the second fastest speed (1.4 body lengths per second), but with a decrease in the rostral tapered region (Fig. 2), increasing the total area under the fin (Fig.9A). An increment in the area under the fin will increase the volume of displaced water and therefore the momentum transfer to the fluid.

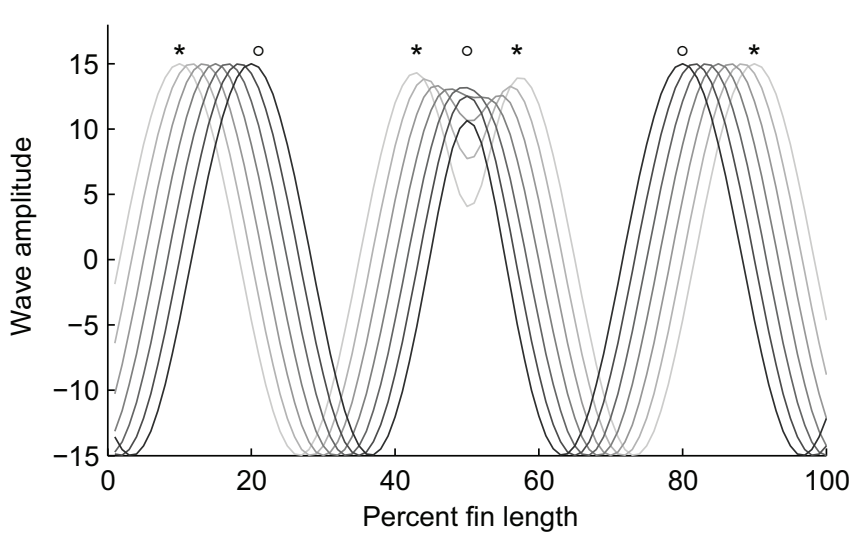

Fig. 11. The basis of variations in the number of undulations along the fin while hovering. As shown in Fig. 6A,C, the number of undulations on the fin forms a sawtooth pattern when hovering. The falling slope of the sawtooth is formed when four waves suddenly become three. As the two undulations approach each other (light gray), four peaks are visible (asterisks). Moments later (dark gray line), two of those peaks overlap and the total number of undulations decreases to three (open circles).

There are many different possible combinations of fin parameters that can result in a given swimming speed. It is worth considering some of the factors that favor certain combinations over others. At slow swimming speeds of less than one body length per second, the cost of transport (energy per unit mass per distance) does not seem to be a primary concern, given that tail waves are generating forces that directly retard motion. In this range of swimming speeds, the generation of enough heave force to counter the fish's negative buoyancy or the precise control of position seem to be more important. At the other extreme of swimming speeds, for approximately two body lengths per second or higher, the fish is likely attempting to generate as much as thrust as possible while minimizing the drag induced by the undulation of the fin, rather than minimize the cost of transport. If so, the lack of influence of cost of transport at high speeds would be similar to the findings of a prior study examining energy use from slow to fast swim speeds (Korsmeyer et al., 2002). That study showed that fish that use median paired-fin swimming for movement at low velocities began to use body-caudal fin swimming at higher speeds, despite its higher cost of transport.

For mid-range swimming speeds (from approximately one to two body lengths per second) it is possible that the fish is trying to minimize the cost of transport. In other work we have found that the cost of transport scales with the cube of amplitude but the square of frequency (Bale et al., 2010) (R. Bale, A. P. S. Bhalla, M.A.M. and N. A. Patankar, manuscript in preparation), which may be why frequency modulation dominates over amplitude modulation at low surge velocities. Blake (Blake, 1983) also suggested that amplitude was relatively unchanging over the swim speeds in his study $(0.2$ to 5 body lengths per second). Across our three highest swimming speeds, over which swim speed more than doubles, the frequency only increases by approximately $0.5 \mathrm{~Hz}$ (from 9.3 to $9.9 \mathrm{~Hz}$ ). This may indicate that $10 \mathrm{~Hz}$ is near the peak continuous activation rate of fin muscle for our subject, forcing the use of energetically less favorable amplitude increases. Although we cannot validate our hypothesis that cost of transport could be a key factor in the selection of the fin parameter regime at intermediate swim speeds from our current experiments, future work using computational fluid dynamics, physical models and energetics experiments on the fish could help to elucidate this hypothesis. 


\section{Implications of kinematics for force magnitude and direction} In prior studies, we have used computational fluid dynamics and a bio-mimetic ribbon fin robot to quantify how force magnitude and direction varies with most of the kinematic variables considered here (Shirgaonkar et al., 2008; Sefati et al., 2012; Curet et al., 2011a; Curet et al., 2011b). It is therefore useful to consider the implications of our kinematics results in light of those findings.

Across the wide range of surge velocities, one kinematic parameter that was largely stable was the number of undulations (Table 1). Our prior computational and robotic studies indicate that the observed number of undulations (two to three) generates maximal thrust (Shirgaonkar et al., 2008; Curet et al., 2011b). Below this number of undulations, although the wave velocity increases, the amount of force in the surge direction decreases (in the limit, with zero undulations, all the force goes into the vertical, or heave, direction). Above this number of undulations, the wave velocity decreases and causes a decline in force.

Robotic and computational work has shown that increases in amplitude increase surge force faster than increases in frequency (Curet et al., 2011b; Shirgaonkar et al., 2008). However, we have also found that the cost of transport scales with the cube of amplitude but the square of frequency (Bale et al., 2010), which may be why frequency modulation appears to be favored over amplitude modulation in the intermediate velocity regime where cost of transport may play a significant role. Blake (Blake, 1983) also reported that amplitude was relatively unchanging over swim speeds in his study.

A possible complication for the fish's control of its speed is that changes in frequency and amplitude of the traveling wave not only change the magnitude of surge force, they also change the direction of the net propulsive force from the fin, which is the sum of the heave and surge forces (Shirgaonkar et al., 2008; Curet et al., 2011a; Curet et al., 2011b). The prior robotic and computational analyses show that as frequency rises, the heave force grows faster than the surge force (Shirgaonkar et al., 2008; Curet et al., 2011b), resulting in an increase in the angle of the net force with respect to the line of fin insertion. Computational analyses also show that increasing amplitude has the opposite effect, with surge force growing faster than heave (Shirgaonkar et al., 2008), therefore decreasing the angle of the net force. If the fish were to try to maintain the same net force direction as it increases swimming speed with increased amplitude or increased frequency, it may need to change the number of undulations (fewer undulations increase heave force), change the body pitch angle, or maintain the same pitch angle and number of undulations but counter-torque around the pitch axis by keeping the pectoral fins held in the flow so as to achieve an additional upward or downward force as needed.

One further factor to consider is traveling wave velocity, $f \lambda$ (where $\lambda$ is fin length over number of undulations). For hovering, and the lowest surge speed, the head and tail waves are similar in velocity. Above $2.6 \mathrm{~cm} \mathrm{~s}^{-1}$, tail waves are faster than head waves. In addition to tail waves being faster than head waves, there is an additional factor that increases the force from tail waves compared with head waves occurring at the same wave velocity. Surge force increases as the difference between the wave velocity and fluid velocity increases. For head waves, this difference in velocity between the fin and ambient fluid is the wave velocity minus the swimming velocity; but for tail waves, the difference is wave velocity plus the swimming velocity. Between their higher velocity and higher force production per length, tail waves at low surge speeds can occur over a shorter length of the fin while still providing sufficient force for controlling position via nodal point control or for countering the body's negative buoyancy.
An indirect measure of swimming efficiency for undulatory swimmers is the ratio of the body velocity to the traveling wave velocity, called the wave efficiency (Gray and Lissmann, 1964; Maladen et al., 2009) or slip (Gillis, 1998; Lighthill, 1975). Typically this measure is estimated when there are unidirectional traveling waves along the body or fin. When we only examine cases with unidirectional traveling waves (swim velocities above $14.9 \mathrm{~cm} \mathrm{~s}^{-1}$ ), we obtain a wave efficiency of $0.5 \pm 0.1$ at a surge velocity of $19.7 \mathrm{~cm} \mathrm{~s}^{-1}$, and a wave efficiency of $0.7 \pm 0.1$ at a surge velocity of $32.6 \mathrm{~cm} \mathrm{~s}^{-1}$ (Table 1 ). A wave efficiency of 0.7 is similar to what is found in the American eel, Anguilla rostrata $[\sim 0.8$ (Gillis, 1998)].

A final force characteristic we will consider is the muscle force needed to stretch the fin when it is undulated. As the angle of separation between the bony rays of the ribbon fin increases, the force needed to stretch the fin also increases. The amount of stretch might not be insignificant: as Fig. 9B shows, the edge of the fin is 1.3 times the length of the fin base for a hovering fish. Although there are no studies on the structure and composition of the interray membrane of the black ghost knifefish, it is most likely composed of a collagenous membrane not unlike the membrane between pectoral fin rays of the bluegill sunfish, which has a Young's modulus in the range of 0.3 to $1 \mathrm{MPa}$ (Lauder and Madden, 2006). Although it is possible that the rest configuration of the fin might have some slack material to facilitate low-amplitude fin ray deflections, for high amplitudes the force needed to stretch the fin could be significant. With the fin membrane $\sim 0.2 \mathrm{~mm}$ thick and $10 \mathrm{~mm}$ tall (M.A.M., unpublished measurements), the stretching force needed could be quite significant in comparison with typical drag (MacIver et al., 2010) and thrust estimates (Shirgaonkar et al., 2008).

\section{CPG model of fin movement}

Although it has not yet been studied in knifefish, it is most likely that the undulatory waves along the fin are generated by coupled CPGs across spinal segments, similar to the generation of undulatory body waves in the lamprey (Cohen and Wallén, 1980; Grillner et al., 1995). The neurally inspired CPG model used here was able to generate kinematic patterns in good agreement with observed behavior. However, as an abstract oscillator model, its utility is not in generating specific physiological predictions, but rather in understanding the dynamics of populations of oscillatory centers (Ijspeert, 2008). This includes, most notably, the generation of inward counter-propagating waves that meet at the nodal point. When two waves of the same frequency, amplitude and wavelength come together traveling in opposite directions along a passive medium, standing waves result. However, consistent with experimental observations, the model generates two inward counterpropagating traveling waves.

In the model, after initial random seeding of the coupling phases ( $\theta_{i}$ and $\theta_{j}$ in Eqn 2), the coupling phases converge to values consistent with simulation parameters (Table 2 , the coupling weights and $C V_{1}, C V_{2}, C R_{1}$ and $C R_{2}$ ). The phase coupling to which the model converges prevents sharp jumps in phase, as would occur if two independent waves were generated. In addition, there is a local minima in drive at the nodal point, resulting in decreased wave amplitude in the region around the nodal point and setting the direction of the waves on either side of the point to be inward toward the point. The switch in drive gradient polarity, a function of the simulation parameters (Table2), prevents each wave from continuing past the nodal point. Prevention of standing waves is necessary because of two problematic mechanical side effects: first, only 
Table 2. Simulation parameters

\begin{tabular}{lcccc}
\hline Simulated swim speed $\left(\mathrm{cm} \mathrm{s}^{-1}\right)$ & $d_{\mathrm{a}}$ & $d_{\mathrm{b}}$ & $d_{\mathrm{c}}$ & $x_{\mathrm{b}}$ \\
\hline 0 & 1.23 & 1.14 & 1.26 & 0.65 \\
2.6 & 1.11 & 1.05 & 1.14 & 0.70 \\
6.1 & 1.50 & 1.47 & 1.50 & 0.85 \\
14.9 & 1.74 & 1.74 & 1.71 & 0.50 \\
19.7 & 2.52 & 2.49 & 2.47 & 0.50 \\
32.6 & 2.64 & 2.64 & 2.58 & 0.50 \\
\hline
\end{tabular}

$d_{\mathrm{a}}$ is the input drive to the oscillators of the most rostral ray, $d_{\mathrm{b}}$ is the drive to the ray at location $x_{b}$ (normalized fin base length) and $d_{c}$ is the drive to the most caudal ray. $x_{\mathrm{a}}$ is fixed at 0.01 (most rostral ray) and $x_{\mathrm{c}}$ is fixed at 1.00 (most caudal ray).

hovering could be achieved, because the mechanism for pushing fluid along the fin - a traveling wave - would not occur, regardless of the position of the nodal point. Second, with standing waves there are five times larger variations in the generated vertical force than when hovering with two traveling waves coming together at a nodal point, potentially compromising the fish's dorsoventral stability (Curet et al., 2011a). Thus the model highlights the need for neuronal mechanisms that provide multiple traveling waves while preventing standing waves, and its structure suggests one possible way to achieve this.

In the model, the number of undulations depends on the differential drive level at different positions along the length of the fin. Waves travel along the fin from rays with high levels of drive to rays with low drive. The larger the difference in drive, the more undulations along the fin. This happens because waves are created more rapidly in one location of the fin than can travel across the low drive regions, increasing the number of undulations along the fin. This provides an experimentally testable hypothesis as to the form of descending spinal control for varying the number of undulations along the fin. Matsushima and Grillner (Matsushima and Grillner, 1992) were able to control the propagation of activity along the spinal cord of a lamprey by applying an excitatory amino acid (NMDA) gradient. Waves traveled away from the location of greatest concentration. The NMDA gradient is comparable to the differential drive of our model and is a possible mechanism by which the fish creates undulatory fin motion.

The differential activation model suggests that the maximum number of undulations on the fin is bounded by the number of fin rays and the maximum difference in drive that can be obtained between a set of rays. The frequency of each ray is dictated by its input drive. We also found that the model predicted a minimum number of undulations along the fin. The fewest waves along the fin occurred by giving the same drive input to all rays. In this condition, there is half of a full wave along the fin, because both ends of the fin are at fixed points. As the drive input to the most rostral rays increases, waves start traveling caudally, while as the drive to the most caudal rays increases, waves start traveling rostrally.

Knifefish are capable of switching swimming direction very rapidly, in approximately one-tenth of a second (Maclver et al., 2001). In the case of our model, after changing the input drive the kinematics of the fin change rapidly and then tend to slowly converge to a steady state. Presumably the knifefish use proprioceptive feedback to adjust the drive signal to different parts of the fin in a more optimal way, reaching the desired state in a much shorter period of time. A feedback controller could be implemented for our model to achieve a similar outcome.

\section{ACKNOWLEDGEMENTS}

We are indebted to Omar Hassan and Uzair Admani for their assistance with fin edge digitization. Many thanks to Victoria Stodden for assistance with the companion website for this paper at http://www.runmycode.org/CompanionSite/ Site194.

\section{AUTHOR CONTRIBUTIONS}

M.A.M., O.M.C. and G.V.L. conceived experiments. O.M.C., G.V.L. and M.A.M. collected data. R.R.T. and M.A.M. performed analyses. M.A.M. and R.R.T. wrote the initial manuscript. All authors contributed to the final manuscript.

\section{FUNDING}

This work was supported by National Science Foundation grants [IOS-0846032 to M.A.M., and CMMI-0941674 to M.A.M. and G.V.L.].

\section{REFERENCES}

Albert, J. S. (2001). Species diversity and phylogenetic systematics of American knifefishes (Gymnotiformes, Teleostei). Misc. Publ. Mus. Zool. Univ. Mich. 190, $1-127$.

Albert, J. S. and Crampton, W. G. R. (2005). Diversity and phylogeny of neotropical electric fishes (Gymnotiformes). In Electroreception, pp. 360-409. New York, NY: Springer.

Bale, R., Hao, M., Bhalla, A. and Patankar, N. (2010). On Gray's paradox and efficiency measures for swimming. In American Physical Society, Division of Fluid Dynamics, 63rd Annual Meeting. Long Beach, CA: Bulletin of the APS.

Blake, R. W. (1983). Swimming in the electric eels and knifefishes. Can. J. Zool. 61, 1432-1441.

Boyer, F., Gossiaux, P. B., Jawad, B., Lebastard, V. and Porez, M. (2012). Model for a sensor inspired by electric fish. IEEE Trans. Robotics 28, 492-505.

Cohen, A. H. and Wallén, P. (1980). The neuronal correlate of locomotion in fish "Fictive swimming" induced in an in vitro preparation of the lamprey spinal cord. Exp. Brain Res. 41, 11-18.

Cowan, N. J. and Fortune, E. S. (2007). The critical role of locomotion mechanics in decoding sensory systems. J. Neurosci. 27, 1123-1128.

Curet, O. M., Maclver, M. A. and Patankar, N. (2009). Multi-directional thrusting using oppositely traveling waves in knifefish swimming. American Physical Society, Division of Fluid Dynamics, 62nd Annual Meeting. Minneapolis, Minnesota. Bulletin of the APS.

Curet, O. M., Patankar, N. A., Lauder, G. V. and Maciver, M. A. (2011a). Aquatic manoeuvering with counter-propagating waves: a novel locomotive strategy. J. $R$. Soc. Interface 8, 1041-1050.

Curet, O. M., Patankar, N. A., Lauder, G. V. and Maclver, M. A. (2011b). Mechanical properties of a bio-inspired robotic knifefish with an undulatory propulsor. Bioinspir. Biomim. 6, 026004

Epstein, M., Colgate, J. E. and Maclver, M. A. (2005). A biologically inspired robotic ribbon fin. Presented at the 2005 IEEE RSJ (IROS) Workshop on Morphology, Control and Passive Dynamics, 2 August 2005, Edmonton, Canada.

Epstein, M., Colgate, J. E. and Maclver, M. A. (2006). Generating thrust with a biologically inspired robotic ribbon fin. 2006 IEEE/RSJ International Conference on Intelligent Robots and Systems, Vols 1-12. 2006. 2412-2417.

Fortune, E. S. (2006). The decoding of electrosensory systems. Curr. Opin. Neurobiol. 16, $474-480$

Gillis, G. B. (1998). Environmental effects on undulatory locomotion in the American eel Anguilla rostrata: kinematics in water and on land. J. Exp. Biol. 201, 949-961.

Gray, J. and Lissmann, H. W. (1964). The locomotion of nematodes. J. Exp. Biol. 41, 135-154.

Grillner, S., Deliagina, T., Ekeberg O, el Manira, A., Hill, R. H., Lansner, A., Orlovsky, G. N. and Wallén, P. (1995). Neural networks that co-ordinate locomotion and body orientation in lamprey. Trends Neurosci. 18, 270-279.

Ijspeert, A. J. (2008). Central pattern generators for locomotion control in animals and robots: a review. Neural Netw. 21, 642-653.

ljspeert, A. J., Crespi, A., Ryczko, D. and Cabelguen, J. M. (2007). From swimming to walking with a salamander robot driven by a spinal cord model. Science $\mathbf{3 1 5}$, 1416-1420.

Jayne, B. C. and Lauder, G. V. (1995). Speed effects on midline kinematics during steady undulatory swimming of largemouth bass. Micropterus salmoides. J. Exp. Biol. 198, 585-602.

Korsmeyer, K. E., Steffensen, J. F. and Herskin, J. (2002). Energetics of median and paired fin swimming, body and caudal fin swimming, and gait transition in parrotfish (Scarus schlegell) and triggerfish (Rhinecanthus aculeatus). J. Exp. Biol. 205, 1253-1263.

Lannoo, M. J. and Lannoo, S. J. (1993). Why do electric fishes swim backwards? An hypothesis based on gymnotiform foraging behavior interpreted through sensory constraints. Environ. Biol. Fishes 36, 157-165.

Lauder, G. V. and Madden, P. G. A. (2006). Learning from fish: Kinematics and experimental hydrodynamics for roboticists. Int. J. Automation Comput. 3, 325-335.

Lighthill, J. (1975). Mathematical Biofluidynamics. Philadelphia, PA: Society for Industrial and Applied Mathematics.

Maclver, M. A., Sharabash, N. M. and Nelson, M. E. (2001). Prey-capture behavior in gymnotid electric fish: motion analysis and effects of water conductivity. J. Exp. Biol. 204, 543-557.

Maclver, M. A., Fontaine, E. and Burdick, J. W. (2004). Designing future underwater vehicles: principles and mechanisms of the weakly electric fish. IEEE J. Oceanic Eng. 29, 651-659.

Maclver, M. A., Patankar, N. A. and Shirgaonkar, A. A. (2010). Energy-information trade-offs between movement and sensing. PLoS Comput. Biol. 6, e1000769. 
Maladen, R. D., Ding, Y., Li, C. and Goldman, D. I. (2009). Undulatory swimming in sand: subsurface locomotion of the sandfish lizard. Science 325, 314-318.

Matsushima, T. and Grillner, S. (1992). Neural mechanisms of intersegmental coordination in lamprey: local excitability changes modify the phase coupling along the spinal cord. J. Neurophysiol. 67, 373-388.

Nanjappa, P., Brand, L. and Lannoo, M. J. (2000). Swimming patterns associated with foraging in phylogenetically and ecologically diverse American weakly electric teleosts (Gymnotiformes). Environ. Biol. Fishes 58, 97-104.

Nelson, M. E. and Maclver, M. A. (2006). Sensory acquisition in active sensing systems. J. Comp. Physiol. A 192, 573-586.

Rose, G. J. and Canfield, J. G. (1993). Longitudinal tracking responses of the weakly electric fish, Sternopygus. J. Comp. Physiol. A 171, 791-798.

Ruiz-Torres, R., Curet, O. M., Lauder, G. V. and Maclver, M. A. (2012). The kinematics of ribbon fin propulsion in a weakly electric knifefish. RunMyCode Companion Website, www.runmycode.org/CompanionSite/Site194.

Sefati, S., Fortune, E. S. and Cowan, N. J. (2010). Counter-propagating waves in the ribbon fin of Eigenmannia virescens enhance maneuverability. Society of
Comparative Biology Annual Meeting, Seattle, WA, USA. http://www.sicb.org/ meetings/2010/schedule/abstractdetails.php3?id=1067.

Sefati, S., Neveln, I., Maclver, M. A., Fortune, E. S. and Cowan, N. J. (2012) Counterpropagating waves enhance maneuverability and stability: a bio-inspired strategy for robotic ribbon-fin propulsion. In Proc. IEEE RAS EMBS Int. Conf. Biomed. Robot. Biomechatron, 24-27 June, pp. 1620-1625. Rome: IEEE.

Shirgaonkar, A. A., Curet, O. M., Patankar, N. A. and Maciver, M. A. (2008). The hydrodynamics of ribbon-fin propulsion during impulsive motion. J. Exp. Biol. 211, 3490-3503.

Snyder, J. B., Nelson, M. E., Burdick, J. W. and Maciver, M. A. (2007). Omnidirectional sensory and motor volumes in electric fish. PLoS Biol. 5, e301.

Solberg, J. R., Lynch, K. M. and Maclver, M. A. (2008). Active electrolocation for underwater target localization. Int. J. Robot. Res. 27, 529-548.

Turner, R. W., Maler, L. and Burrows, M. (ed.) (1999). Electroreception and Electrocommunication. Vol. 202, pp. 1167-1458. Cambridge: The Journal of Experimental Biology. 